\title{
CLASSIFYING HOPF ALGEBRAS OF A GIVEN DIMENSION
}

\author{
MARGARET BEATTIE AND GASTÓN ANDRÉS GARCÍA
}

\begin{abstract}
Classifying all Hopf algebras of a given finite dimension over $\mathbb{C}$ is a challenging problem which remains open even for many small dimensions, not least because few general approaches to the problem are known. Some useful techniques include counting the dimensions of spaces related to the coradical filtration [Fu2, [AN1, [BD], studying sub- and quotient Hopf algebras [G], GV], especially those sub-Hopf algebras generated by a simple subcoalgebra [N5], working with the antipode Ng1, Ng2, Ng3, Ng4, and studying Hopf algebras in Yetter-Drinfeld categories to help to classify Radford biproducts ChNg. In this paper, we add to the classification tools in $[\mathrm{BG}$ and apply our results to Hopf algebras of dimension $r p q$ and $8 p$ where $p, q, r$ are distinct primes. At the end of this paper we summarize in a table the status of the classification for dimensions up to 100 to date.
\end{abstract}

\section{INTRODUCTION}

Let $\mathbb{k}$ be an algebraically closed field of characteristic 0 . The question of classifying all Hopf algebras of a given dimension over $\mathbb{k}$ goes back to Kaplansky in 1975. To date, there are very few general results. The Kac-Zhu Theorem [Z], states that a Hopf algebra of prime dimension is isomorphic to a group algebra. S.-H. Ng $[\mathrm{Ng} 1]$ proved that in dimension $p^{2}$, the only Hopf algebras are the group algebras and the Taft algebras, using previous results in [AS1, Mas3]. It is a common belief that a Hopf algebra of dimension $p q$, where $p$ and $q$ are distinct prime numbers, is semisimple. Hence, it should be isomorphic to a group algebra or a dual group algebra by [EGel1, GelW], Mas5], [N2], [So. This conjecture has been verified for some particular values of $p$ and $q$, see AN1, BD, EGel3, Ng2, Ng3, Ng4. Hilgemann and S.-H. Ng gave the classification of Hopf algebras of dimension $2 p^{2}$ in [HNg] and more recently Cheng and $\mathrm{Ng} \mathrm{ChNg}$ studied the case $4 p$, solving the problem for dimension 20,28 and 44 .

In fact, all Hopf algebras of dimension $\leq 23$ are classified: for dimension $\leq 11$ the problem was solved by [W]; an alternative proof appears in [S]. The classification for dimension 12 was done by F in the semisimple case and then completed by [N5 in the general case and for dimension 16 it was solved by $[\mathrm{K}, \mathrm{CDR}, \mathrm{B} 2, \mathrm{CDMM}$ and GV]. For dimension 18 the problem was solved by D. Fukuda [Fu1] and recently Cheng and Ng finished the classification for dimension 20. For the state of the classification of low dimensional Hopf algebras as of 2009, see [B3].

The classification appears more difficult for even dimensions as studied in this article. One reason may be that for $H$ a nonsemisimple Hopf algebra of odd dimension, either $H$ or $H^{*}$ has a nontrivial grouplike element. The smallest dimension that is still unclassified is 24 and, since the classification for dimension 27 was recently completed in [BG], the next unclassified dimension after 24 is 32 .

In this paper we study Hopf algebras over $\mathbb{k}$ whose dimension is either smaller than 100 or can be decomposed into the product of a small number of prime numbers. In particular, we give some partial results on Hopf algebras of dimension $8 p$, with applications to the case of dimension 24, and dimension $r p q$, where $r, p, q$ are distinct prime numbers. Since there are many results on the classification problem for dimension $4 p \mathrm{ChNg}$ but the complete classification is incomplete, we cannot hope to complete the classification for dimension $8 p$. However we can narrow the possibilities.

We will say that a Hopf algebra $H$ is of type $(r, s)$ if $|G(H)|=r$ and $\left|G\left(H^{*}\right)\right|=s$.

Date: December 19, 2014.

2010 Mathematics Subject Classification. 16T05.

This work was partially supported by ANPCyT-Foncyt, CONICET, Ministerio de Ciencia y Tecnología (Córdoba) and Secyt (UNC). 
Theorem A. Let $H$ be a nonsemisimple Hopf algebra of dimension $8 p$ with $p$ an odd prime. If $H$ is not of type $(r, s)$ with $r, s$ powers of $2,(2 p, 2)$ or $(2 p, 4)$, then $H$ is pointed or basic.

Using counting arguments we can improve the theorem above in case $p=3$.

Theorem B. Let $H$ be a Hopf algebra of dimension 24 such that the coradical is not a sub-Hopf algebra of $H$. Then $H$ is of type $(2,2),(2,4)$ or $(6,4)$.

\section{ACKNOWLEDGEMENTS}

This version of the paper contains a correction on the published version. The statement and proof of Proposition 2.17 are changed and the proof of the results that follow from it are corrected accordingly. We thank H.-S. Ng for kindly communicating the gap to us and for the careful reading of our paper.

\section{Preliminaries}

In this section we introduce notation, recall some previous results which help with the classification of finite dimensional Hopf algebras, see [AN1], [N5], [S], BD], [B2], GV], [Fu1], and introduce a few new ones. For the general theory of Hopf algebras see [M], [S].

2.1. Conventions. Throughout this paper $p, q$ will denote odd prime numbers, $C_{k}$ the cyclic group of order $k$ and $\mathbb{D}_{k}$ the dihedral group of order $2 k$. Unless otherwise specified, all Hopf algebras in this article are finite dimensional over a field $\mathbb{k}$ algebraically closed of characteristic zero.

Remark 2.1. By $[\mathrm{LR}$, with the assumptions above, a Hopf algebra is semisimple if and only if it is cosemisimple if and only if $S^{2}$, the square of the antipode, is the identity. Thus if $L, K$ are semisimple sub-Hopf algebras of a Hopf algebra $H$, then $\langle L, K\rangle$, the sub-Hopf algebra of $H$ generated by $L$ and $K$ is semisimple since $S_{H}^{2}$ is the identity on $L$ and on $K$.

For $H$ a Hopf algebra over $\mathbb{k}$ then $\Delta, \varepsilon, S$ denote respectively the comultiplication, the counit and the antipode; $G(H)$ denotes the group of grouplike elements of $H ; H_{0}$ denotes the coradical; $\left(H_{n}\right)_{n \in \mathbb{N}}$ denotes the coradical filtration of $H$ and $L_{h}$ (resp. $R_{h}$ ) is the left (resp. right) multiplication in $H$ by $h$. We say that $H$ is pointed if $H_{0}=\mathbb{k} G(H)$.

The set of $(h, g)$-primitives (with $h, g \in G(H))$ and set of skew-primitives of $H$ are:

$$
\begin{aligned}
\mathcal{P}_{h, g}(H) & :=\{x \in H \mid \Delta(x)=x \otimes h+g \otimes x\}, \\
\mathcal{P}(H) & :=\sum_{h, g \in G(H)} \mathcal{P}_{h, g}(H) .
\end{aligned}
$$

We say that $x \in \mathbb{k}(h-g)$ is a trivial skew-primitive; a skew-primitive not contained in $\mathbb{k} G(H)$ is nontrivial.

Let $\mathcal{M}^{*}(n, \mathbb{k})$ denote the simple coalgebra of dimension $n^{2}$, dual to the matrix algebra $\mathcal{M}(n, \mathbb{k})$. We say that a coalgebra $C$ is a $d \times d$ matrix-like coalgebra if $C$ is spanned by elements $\left(e_{i j}\right)_{1 \leq i, j \leq n}$ such that $\Delta\left(e_{i j}\right)=\sum_{1 \leq l<n} e_{i l} \otimes e_{l j}$ and $\varepsilon\left(e_{i j}\right)=\delta_{i j}$. If the set $\left(e_{i j}\right)_{1 \leq i, j \leq d}$ of a coalgebra $C$ of dimension $d^{2}$ is linearly independent, following Ştefan we call $\mathbf{e}=\left\{e_{i j}: 1 \leq i, j \leq d\right\}$ a multiplicative matrix and then $C \simeq \mathcal{M}^{*}(d, \mathbb{k})$ as coalgebras.

Since the only semisimple and pointed Hopf algebras are the group algebras, we shall adopt the convention that 'pointed' means 'pointed nonsemisimple'. Similarly, we say that a finite dimensional Hopf algebra $H$ is basic if $H$ is basic as an algebra or copointed as a coalgebra, i.e., all simple $H$ modules are one-dimensional or the dual is pointed, but $H$ is not the dual of a group algebra.

Recall that a tensor category $\mathcal{C}$ over $\mathbb{k}$ has the Chevalley property if the tensor product of any two simple objects is semisimple. We shall say that a Hopf algebra $H$ has the Chevalley property if the category Comod $(H)$ of $H$-comodules does.

Remarks 2.2. (i) The notion of the Chevalley property in the setting of Hopf algebras was introduced by AEGel]: it is said in loc. cit. that a Hopf algebra has the Chevalley property if the category $\operatorname{Rep}(H)$ of $H$-modules does. 
(ii) Unlike AEGel, in CDMM, Section 1], the authors refer to the Chevalley property in the category of $H$-comodules; this definition is the one we adopt. Note that it is equivalent to say that the coradical $H_{0}$ of $H$ is a sub-Hopf algebra.

(iii) If $H$ is semisimple or pointed then it has the Chevalley property.

Let $N$ be a positive integer and let $q$ be a primitive $N^{t h}$ root of unity. We denote by $T_{q}$ the Taft algebra which is generated as an algebra by the elements $g$ and $x$ satisfying the relations $x^{N}=0=$ $1-g^{N}, g x=q x g$. Taft Hopf algebras are self-dual and pointed of dimension $N^{2}$ with $g$ grouplike and $x$ a $(1, g)$-primitive, i.e., $\Delta(g)=g \otimes g$ and $\Delta(x)=x \otimes 1+g \otimes x$. If $N=2$ so that $q=-1$, then $T_{-1}$ is called the Sweedler Hopf algebra and will be denoted $H_{4}$ thoughout this article.

2.2. Spaces of coinvariants. Let $K$ be a coalgebra with a distinguished grouplike 1 . If $M$ is a right $K$-comodule via $\delta$, then the space of right coinvariants is

$$
M^{\operatorname{co} \delta}=\{x \in M \mid \delta(x)=x \otimes 1\} .
$$

Left coinvariants are defined analogously. If $\pi: H \rightarrow K$ is a morphism of Hopf algebras, then $H$ is a right $K$-comodule via $(1 \otimes \pi) \Delta$. In this case $H^{\operatorname{co} \pi}:=H^{\operatorname{co}(1 \otimes \pi) \Delta}$ and $H^{c o \pi}$ is a subalgebra of $H$.

We make the following observation.

Lemma 2.3. Let $\pi: H \rightarrow K$ be a Hopf algebra map and let $R:=H^{c o \pi}$. Then $\left.\pi\right|_{R}=\left.\varepsilon\right|_{R}$.

Proof. Let $z \in H^{c o \pi}$. Since $\pi$ is a morphism of Hopf algebras,

$$
\Delta_{K} \pi(z)=(\pi \otimes \pi) \Delta_{H}(z)=\pi\left(z_{1}\right) \otimes \pi\left(z_{2}\right)=\pi(z) \otimes 1,
$$

so that, applying $m_{K} \circ\left(\varepsilon_{K} \otimes \mathrm{id}_{K}\right)$ to the equation above, we obtain $\pi(z)=\varepsilon_{K}(\pi(z)) \in \mathbb{k}$. Again, since $\pi$ is a Hopf algebra map, $\varepsilon_{K}(\pi(z))=\varepsilon_{H}(z)$ and so $\pi(z)=\varepsilon_{H}(z)$.

2.3. Extensions of Hopf algebras. Recall [AD that an exact sequences of Hopf algebras is a sequence of Hopf algebra morphisms $A \stackrel{\imath}{\hookrightarrow} H \stackrel{\pi}{\rightarrow} B$ where $A, H, B$ are any Hopf algebras, $\imath$ is injective, $\pi$ is surjective, $\pi \imath=\varepsilon_{A}$, $\operatorname{ker} \pi=A^{+} H$ and $A=H^{c o \pi}$. An exact sequence is called central if $A$ is contained in the centre of $H$.

The next result will be useful throughout. For a proof see [GV, Lemma 2.3].

Lemma 2.4. If $\pi: H \rightarrow B$ is an epimorphism of Hopf algebras then $\operatorname{dim} H=\operatorname{dim} H^{\operatorname{co} \pi} \operatorname{dim} B$. Moreover, if $A=H^{\mathrm{co} \pi}$ is a sub-Hopf algebra of $H$ then the sequence $A \stackrel{\imath}{\hookrightarrow} H \stackrel{\pi}{\rightarrow} B$ is exact.

The following proposition tells us how to compute, in a particular case, the dimension of the coradical of $H^{*}$ using exact sequences.

Proposition 2.5. Let $\Gamma$ be a finite group and $A \hookrightarrow H \rightarrow \mathbb{k} \Gamma$ an exact sequence of Hopf algebras. Then $\operatorname{dim}\left(H^{*}\right)_{0}=\operatorname{dim}(H / \operatorname{rad} H)=|\Gamma| \operatorname{dim}\left(A^{*}\right)_{0}$.

Proof. The statement follows from the proof of [GV, Lemma 5.9]. The idea is the following: since the sequence is exact, $H$ is the $\Gamma$-crossed product $A * \Gamma$. Let $g \in \Gamma$, then the weak action of $g$ on $A$ defines an algebra map and consequently $\operatorname{rad} A$ is stable by $\Gamma$. Then $\operatorname{rad} A * \Gamma$ is a nilpotent ideal of $A * \Gamma$ and $\operatorname{rad} A * \Gamma \subseteq \operatorname{rad} H$. Since $H /(\operatorname{rad} A * \Gamma)$ is semisimple, it follows that $\operatorname{rad} H \subseteq \operatorname{rad} A * \Gamma$ and hence $\operatorname{dim}\left(H^{*}\right)_{0}=\operatorname{dim}(H / \operatorname{rad} H)=\operatorname{dim}(A * \Gamma / \operatorname{rad} A * \Gamma)=|\Gamma| \operatorname{dim}(A / \operatorname{rad} A)=|\Gamma| \operatorname{dim}\left(A^{*}\right)_{0}$.

2.4. Yetter-Drinfel'd modules. For $H$ any Hopf algebra, a left Yetter-Drinfeld module $M$ over $H$ is a left $H$-module $(M, \cdot)$ and a left $H$-comodule $(M, \delta)$ such that for all $h \in H, m \in M$,

$$
\delta(h \cdot m)=h_{1} m_{(-1)} \mathcal{S}\left(h_{3}\right) \otimes h_{2} \cdot m_{(0)},
$$

where $\delta(m)=m_{(-1)} \otimes m_{(0)}$. We will denote this category by ${ }_{H}^{H} \mathcal{Y} \mathcal{D}$. 
2.5. On the coradical filtration. We begin by recalling a description of the coradical filtration due to Nichols. More detail can be found in AN1, Section 1].

Let $D$ be a coalgebra over $\mathbb{k}$. Then there exists a coalgebra projection $\pi: D \rightarrow D_{0}$ from $D$ to the coradical $D_{0}$ with kernel $I$, see [M, 5.4.2]. Define the maps

$$
\rho_{L}:=(\pi \otimes \mathrm{id}) \Delta: D \rightarrow D_{0} \otimes D \quad \text { and } \quad \rho_{R}:=(\mathrm{id} \otimes \pi) \Delta: D \rightarrow D \otimes D_{0},
$$

and let $P_{n}$ be the sequence of subspaces defined recursively by

$$
\begin{aligned}
& P_{0}=0, \\
& P_{1}=\left\{x \in D: \Delta(x)=\rho_{L}(x)+\rho_{R}(x)\right\}=\Delta^{-1}\left(D_{0} \otimes I+I \otimes D_{0}\right), \\
& P_{n}=\left\{x \in D: \Delta(x)-\rho_{L}(x)-\rho_{R}(x) \in \sum_{1 \leq i \leq n-1} P_{i} \otimes P_{n-i}\right\}, \quad n \geq 2 .
\end{aligned}
$$

Then by a result of Nichols, $P_{n}=D_{n} \cap I$ for $n \geq 0$, see [AN1, Lemma 1.1]. Suppose that $D_{0}=\bigoplus_{\tau \in \mathcal{I}} D_{\tau}$, where the $D_{\tau}$ are simple coalgebras of dimension $d_{\tau}^{2}$. Any $D_{0}$-bicomodule is a direct sum of simple $D_{0}$-sub-bicomodules and every simple $D_{0}$-bicomodule has coefficient coalgebras $D_{\tau}, D_{\gamma}$ and has dimension $d_{\tau} d_{\gamma}=\sqrt{\operatorname{dim} D_{\tau} \operatorname{dim} D_{\gamma}}$ for some $\tau, \gamma \in \mathcal{I}$, where $d_{\tau}, d_{\gamma}$ are the dimensions of the associated comodules of $D_{\tau}$ and $D_{\gamma}$, respectively.

Now suppose $H$ is a Hopf algebra. Then $H_{n}, P_{n}$ are $H_{0}$-sub-bicomodules of $H$ via $\rho_{R}$ and $\rho_{L}$. As in [AN1], [Fu1], for all $n \geq 1$ we denote by $P_{n}^{\tau, \gamma}$ the isotypic component of the $H_{0}$-bicomodule of $P_{n}$ of type the simple bicomodule with coalgebra of coefficients $D_{\tau} \otimes D_{\gamma}$. If $D_{\tau}=\mathbb{k} g$ for $g$ a grouplike, we use the superscript $g$ instead of $\tau$. If the simple subcoalgebras are $S\left(D_{\tau}\right), S\left(D_{\gamma}\right)$, (respectively $g D_{\tau}$, $D_{\tau} g$ for $g$ grouplike) we write $P_{n}^{S \tau, S \gamma}$,(respectively $P_{n}^{g \tau, g \gamma}, P_{n}^{\tau g, \gamma g}$.) For $D_{\tau}, D_{\gamma}$ simple coalgebras we denote $P^{\tau, \gamma}=\sum_{n \geq 0} P_{n}^{\tau, \gamma}$.

Similarly, for $\Gamma$ a set of grouplikes of $H$, let $P^{\Gamma, \Gamma}$ denote $\sum_{g, h \in \Gamma} P^{g, h}$ and let $H^{\Gamma, \Gamma}:=P^{\Gamma, \Gamma} \oplus \mathbb{k} \Gamma$. If $\mathcal{D}, \mathcal{E}$ are sets of simple subcoalgebras, let $P^{\mathcal{D}, \mathcal{E}}$ denote $\sum_{D \in \mathcal{D}, E \in \mathcal{E}} P^{D, E}$. Since $H_{n}=H_{0} \oplus P_{n}$, we have that $H=H_{0} \oplus \sum_{\tau, \gamma} P^{\tau, \gamma}$.

Following D. Fukuda, we say that the subspace $P_{n}^{\tau, \gamma}$ is nondegenerate if $P_{n}^{\tau, \gamma} \nsubseteq P_{n-1}$. The following results are due to D. Fukuda; note that (ii) is a generalization of [AN1, Cor. 1.3] for $n>1$.

Lemma 2.6. (i) [Fu2, Lemma 3.2] If the subspace $P_{n}^{\tau, \gamma}$ is nondegenerate for some $n>1$, then there exists a set of simple coalgebras $\left\{D_{1}, \cdots, D_{n-1}\right\}$ with $P_{i}^{\tau, D_{i}}, P_{n-i}^{D_{i}, \gamma}$ nondegenerate for all $1 \leq i \leq n$.

(ii) [Fu2, Lemma 3.5] For $S$ the antipode in the Hopf algebra $H$ and $g \in G(H)$,

$$
\operatorname{dim} P_{n}^{\tau, \gamma}=\operatorname{dim} P_{n}^{S \gamma, S \tau}=\operatorname{dim} P_{n}^{g \tau, g \gamma}=\operatorname{dim} P_{n}^{\tau g, \gamma g} .
$$

(iii) [Fu2, Lemma 3.8] Let $C, D$ be simple subcoalgebras such that $P_{m}^{C, D}$ is nondegenerate. If $\operatorname{dim} C \neq$ $\operatorname{dim} D$ or $\operatorname{dim} P_{m}^{C, D}-P_{m-1}^{C, D} \neq \operatorname{dim} C$ then there exists a simple subcoalgebra $E$ such that $P_{\ell}^{C, E}$ is nondegenerate for some $\ell \geq m+1$.

The following facts about dimensions from [AN1] will be useful later.

Lemma 2.7. AN1] Let $H$ be a Hopf algebra with $G:=G(H)$. Then for $n \geq 0, d \geq 1,|G|$ divides $\operatorname{dim} H_{n}$ and $\operatorname{dim} H_{0, d}$, where $H_{0, d}$ denotes the direct sum of the simple subcoalgebras of $H$ of dimension $d^{2}$. Also $H_{n}=H_{0} \oplus P_{n}$ so that $|G|$ divides $\operatorname{dim} P_{n}$.

It is well-known (see for example [AN1]) that if a Hopf algebra $H$ has a nontrivial skew primitive element, then $\operatorname{dim} H$ must be divisible by a square. More precisely we have the following lemma.

Lemma 2.8. Let $H$ be a Hopf algebra with $|G(H)|=m$ and $\operatorname{dim} H=m n$ where $m$, $n$ are relatively prime. Then $H$ has no nontrivial skew-primitive element. 
Proof. Suppose that $x$ is a nontrivial skew-primitive element in $H$ and let $L$ be the sub-Hopf algebra of $H$ generated by $x$ and $G(H)$. By [M, 5.5.1], $L$ is pointed. By [AN1, Proposition 1.8], $\operatorname{dim} L$ is divisible by $r m$ where $r \neq 1$ is a positive integer dividing $m$. Then $\operatorname{dim} H=m n$ is divisible by $r m$, contradicting the fact that $(m, n)=1$.

The next proposition generalizes results of Beattie and Dăscălescu [BD] and gives a lower bound for the dimension of a finite dimensional Hopf algebra without nontrivial skew-primitive elements.

Proposition 2.9. BG, Proposition 3.2] Let $H$ be a non-cosemisimple Hopf algebra with no nontrivial skew-primitives.

(i) For any $g \in G(H)$ there exists a simple subcoalgebra $C$ of $H$ of dimension $>1$ such that $P_{1}^{C, g} \neq 0$, $P_{k}^{C, D}$ is nondegenerate for some $k>1$ and $D$ a simple subcoalgebra of the same dimension as $C$, and $P_{m}^{g, h}$ is nondegenerate for some $m>1$ and $h$ grouplike.

(ii) Suppose $H_{0} \simeq \mathbb{k} G \oplus \sum_{i=1}^{t} \mathcal{M}^{*}\left(n_{i}, \mathbb{k}\right)$ with $t \geq 1,2 \leq n_{1} \leq \ldots \leq n_{t}$. Then

$$
\operatorname{dim} H \geq \operatorname{dim}\left(H_{0}\right)+\left(2 n_{1}+1\right)|G|+n_{1}^{2} .
$$

2.6. Matrix-like coalgebras. The next theorem due to Ştefan has been a key component for several classification results.

Theorem 2.10. [S, Thm. 1.4] Let $D$ be the simple coalgebra $\mathcal{M}^{*}(2, \mathbb{k})$.

(i) For $f$ an antiautomorphism of $D$ such that $\operatorname{ord}\left(f^{2}\right)=n<\infty$ and $n>1$, there exist a multiplicative matrix $\boldsymbol{e}$ in $D$ and a root of unity $\omega$ of order $n$ such that

$$
f\left(e_{12}\right)=\omega^{-1} e_{12}, \quad f\left(e_{21}\right)=\omega e_{21}, \quad f\left(e_{11}\right)=e_{22}, \quad f\left(e_{22}\right)=e_{11} .
$$

(ii) For $f$ be an automorphism of $D$ of finite order $n$, there exist a multiplicative matrix $e$ on $D$ and a root of unity $\omega$ of order $n$ such that $f\left(e_{i j}\right)=\omega^{i-j} e_{i j}$.

Now we recall some useful results on matrix-like coalgebras. In BD all $2 \times 2$ matrix-like coalgebras of dimension less than 4 were described; we summarize in the following theorem.

Theorem 2.11. [BD, Thm. 2.1] Let $D$ be a $2 \times 2$ matrix-like coalgebra of dimension less than 4 . If $\operatorname{dim} D=1,2$ then $D$ has a basis of grouplike elements. If $\operatorname{dim} D=3$, then $D$ has a basis $\{g, h, x\}$ where $g, h$ are grouplike and $x$ is $(g, h)$-primitive.

We end this section with the following lemma.

Lemma 2.12. Let $\pi: H \rightarrow H_{4}$ be a Hopf algebra epimorphism. If $H$ is generated by a simple subcoalgebra $D$ of dimension 4 , then $\operatorname{dim}{ }^{\operatorname{co} \pi} D \geq 2$ or $\operatorname{dim} D^{\text {co } \pi} \geq 2$.

Proof. Since $H_{4}$ is pointed, $\operatorname{dim} \pi(D)<4$. Moreover, since $D$ generates $H$ as an algebra, $\pi(D)$ generates $H_{4}$. Then by Theorem [2.11, $\operatorname{dim} \pi(D)=3$. Let $\left\{e_{i j}\right\}_{1 \leq i, j \leq 2}$ be a multiplicative matrix of $D$, then $\left\{\pi\left(e_{i j}\right)\right\}_{1 \leq i, j \leq 2}$ is a linearly dependent set.

As in the proof of Theorem 2.11, see [BD, Thm. 2.1], we divide the proof into two cases.

Case 1: The set $\left\{\pi\left(e_{11}\right), \pi\left(e_{12}\right), \pi\left(e_{21}\right)\right\}$ is linearly independent.

Then $\pi\left(e_{22}\right)=\pi\left(e_{11}\right)+a \pi\left(e_{12}\right)+b \pi\left(e_{21}\right)$ for scalars $a, b$. By comparing $\Delta \pi\left(e_{22}\right)$ and $\pi \otimes \pi \circ \Delta\left(e_{22}\right)$ one sees that $a b=-1$ so that there exists $0 \neq b \in \mathbb{k}$ such that $\pi\left(e_{22}\right)=\pi\left(e_{11}\right)+b \pi\left(e_{12}\right)-\frac{1}{b} \pi\left(e_{21}\right)$. Then it is straightforward to verify that the linearly independent elements $h_{1}=\pi\left(e_{11}\right)-\frac{1}{b} \pi\left(e_{21}\right)$ and $h_{2}=\pi\left(e_{11}\right)+b \pi\left(e_{12}\right)$ are grouplike.

Suppose that $h_{1}=1$. Then it is straightforward to show that $t_{1}=e_{11}-\frac{1}{b} e_{21} \in{ }^{\text {co } \pi} D$. 
Let $s_{1}=e_{22}-b e_{12}$ and note that $s_{1}, t_{1}$ are linearly independent. Then $\pi\left(s_{1}\right)=\pi\left(e_{22}\right)-b \pi\left(e_{12}\right)=$ $\pi\left(e_{11}\right)+b \pi\left(e_{12}\right)-\frac{1}{b} \pi\left(e_{21}\right)-b \pi\left(e_{12}\right)=\pi\left(e_{11}\right)-\frac{1}{b} \pi\left(e_{21}\right)=\pi\left(t_{1}\right)=1$. Then a computation similar to that for $t_{1}$, shows that $s_{1} \in{ }^{\text {co } \pi} D$. Thus $\operatorname{dim}{ }^{\text {co } \pi} D \geq 2$.

If $h_{2}=1$ then define $t_{2}=e_{11}+b e_{12}$ and $s_{2}=e_{22}+\frac{1}{b} e_{21}$. A computation similar to that above shows that $t_{2}, s_{2} \in D^{c o \pi}$ so that $D^{c o \pi}$ has dimension at least 2 .

Case 2: The set $\left\{\pi\left(e_{11}\right), \pi\left(e_{12}\right), \pi\left(e_{22}\right)\right\}$ is linearly independent.

Then there exist $a, b \in \mathbb{k}$ such that $\pi\left(e_{21}\right)=a \pi\left(e_{11}\right)+b \pi\left(e_{12}\right)-a \pi\left(e_{22}\right)$. If $a \neq 0$, then the case reduces to Case 1. If $a=0$, then $\pi\left(e_{21}\right)=b \pi\left(e_{12}\right)$ and by comparing $\Delta \pi\left(e_{21}\right)$ and $\Delta \pi\left(b e_{12}\right)$, we see that $b=0$ so that $\pi\left(e_{21}\right)=0$. Thus $\Delta\left(\pi\left(e_{11}\right)\right)=\pi\left(e_{11}\right) \otimes \pi\left(e_{11}\right), \Delta\left(\pi\left(e_{22}\right)\right)=\pi\left(e_{22}\right) \otimes \pi\left(e_{22}\right)$ and $\Delta\left(\pi\left(e_{12}\right)\right)=\pi\left(e_{11}\right) \otimes \pi\left(e_{12}\right)+\pi\left(e_{12}\right) \otimes \pi\left(e_{22}\right)$, which implies that $G\left(H_{4}\right)=\left\langle\pi\left(e_{11}\right), \pi\left(e_{22}\right)\right\rangle$. If $\pi\left(e_{11}\right)=1$, then $e_{11} \in D^{\text {co } \pi}$, for

$$
(1 \otimes \pi) \Delta\left(e_{11}\right)=(1 \otimes \pi)\left(e_{11} \otimes e_{11}+e_{12} \otimes e_{21}\right)=e_{11} \otimes \pi\left(e_{11}\right)+e_{12} \otimes \pi\left(e_{21}\right)=e_{11} \otimes 1 .
$$

Moreover, the element $e_{11}+e_{21} \in D^{\mathrm{co} \pi}$, since

$$
\begin{aligned}
(1 \otimes \pi) \Delta\left(e_{11}+e_{21}\right) & =(1 \otimes \pi)\left(e_{11} \otimes e_{11}+e_{12} \otimes e_{21}+e_{21} \otimes e_{11}+e_{22} \otimes e_{21}\right. \\
& =e_{11} \otimes \pi\left(e_{11}\right)+e_{12} \otimes \pi\left(e_{21}\right)+e_{21} \otimes \pi\left(e_{11}\right)+e_{22} \otimes \pi\left(e_{21}\right)=e_{11} \otimes 1+e_{21} \otimes 1 .
\end{aligned}
$$

Thus, $\operatorname{dim} D^{\text {co } \pi} \geq 2$. The case $\pi\left(e_{22}\right)=1$ is completely analogous and implies that $\operatorname{dim}{ }^{\text {co } \pi} D \geq 2$, taking the elements $e_{22}$ and $e_{22}+e_{21}$.

Note that if $D$ in Lemma 2.12 is stable under $S^{2}$ then the proof can be simplified considerably. For then we may choose a multiplicative matrix for $D$ consisting of eigenvectors for $S^{2}$ and we must have that $\pi\left(e_{i i}\right) \in \mathbb{k} C_{2} \subset H_{4}$.

2.7. Hopf algebras generated by a simple subcoalgebra. In this subsection we summarize some known facts about Hopf algebras generated by simple subcoalgebras of dimension 4 . The most important is the next proposition, due to Natale but derived from a result of Ştefan [S].

Proposition 2.13. [N5, Prop. 1.3]. Suppose that $H$ is nonsemisimple Hopf algebra generated by a simple subcoalgebra of dimension 4 which is stable by the antipode. Then $H$ fits into a central exact sequence $\mathbb{k}^{G} \stackrel{\imath}{\hookrightarrow} H \stackrel{\pi}{\rightarrow} A$, where $G$ is a finite group and $A^{*}$ is a pointed nonsemisimple Hopf algebra.

We have the following useful results from [GV]. If $H$ is a Hopf algebra, $\mathcal{Z}(H)$ denotes its centre.

Lemma 2.14. GV, Lemma 4.2] Let $\pi: H \rightarrow K$ be a morphism of Hopf algebras such that $\pi(g)=1$ for some $g \in G(H), g \neq 1$. Suppose that $H$ is generated by a simple subcoalgebra of dimension 4 stable by $L_{g}$. Then $\pi(H) \subseteq \mathbb{k} G(K)$.

The same holds with $R_{g}$ instead of $L_{g}$; or with $\operatorname{ad}_{\ell}(g)$ or $\operatorname{ad}_{r}(g)$ if $g \notin \mathcal{Z}(H)$.

Lemma 2.15. [GV, Lemma 4.3] Let $\pi: H \rightarrow K$ be an epimorphism of Hopf algebras and assume that $K$ is nonsemisimple. Suppose that $H$ is generated by a simple subcoalgebra of $H$ of dimension 4 stable by $S_{H}^{2}$. Then ord $S_{H}^{2}=\operatorname{ord} S_{K}^{2}$.

Remark 2.16. ( $i$ ) If $H$ is generated as an algebra by $C \oplus D$ with $S(C)=D$, then $C$ also generates $H$ as an algebra, since the sub-bialgebra generated by $C$ is finite dimensional and thus a sub-Hopf algebra.

(ii) Suppose that $H$ is generated by a simple subcoalgebra $C$ of dimension 4 stable by $S_{H}^{2}$. If $H_{4}$ is a sub-Hopf algebra of $H^{*}$, then $S_{H}^{4}=\mathrm{id}$. For the inclusion $H_{4} \hookrightarrow H^{*}$ induces a Hopf algebra surjection $H \rightarrow H_{4}$ and by Lemma 2.15 the claim follows. 
We end this section with the following proposition.

Proposition 2.17. Let $\pi: H \rightarrow A$ be a Hopf algebra epimorphism and assume $\operatorname{dim} H=2 \operatorname{dim} A$. Then $H^{\mathrm{co} \pi}=\mathbb{k}\{1, x\}$ with $x$ a $(1, g)$-primitive element with $\pi(x)=0, g \in G(H)$ and ord $g=2 n$ with $n \geq 1$. If $x$ is trivial, i.e., $x \in \mathbb{k} G(H)$, then $H$ fits into an exact sequence of Hopf algebras $\mathbb{k} C_{2} \hookrightarrow H \rightarrow A$. Otherwise $x^{2}=0, x g=-g x$ and $x, g$ generate a pointed sub-Hopf algebra of dimension $4 n$; in particular, $4 \mid \operatorname{dim} H$.

Proof. The statement follows from the proof of $\mathrm{HNg}$, Prop. 1.3]. We reproduce part of the proof. Let $R=H^{\text {co } \pi}$; it is known that $R$ is a left coideal subalgebra and $\operatorname{dim} R=2$. Let $x \in R \backslash\{0\}$ such that $\varepsilon(x)=0$. Then $R=\mathbb{k}\{1, x\}$ and $\Delta(x)=a \otimes 1+b \otimes x$ for some $a, b \in H$. Since $x \in R$, it follows that $x=a$ and $\pi(x)=0$. The coassociativity of $\Delta$ implies that $b$ is grouplike. Denote $g=b$, then $x \in P_{1, g}$ and $x$ is a skew-primitive element. If $x=\alpha(1-g)$ for some $\alpha \in \mathbb{k}^{*}$, then $g^{2}=1$ and $R \simeq \mathbb{k} C_{2}$ implying that $H$ fits into an exact sequence of Hopf algebras $\mathbb{k} C_{2} \hookrightarrow H \rightarrow A$.

Assume $x$ is non-trivial and let $m=\operatorname{ord} g$. As $R$ is a subalgebra stable by the adjoint action of $g$, it follows that $g x g^{-1}=\beta x$ with $\beta^{m}=1$ and $\Delta\left(x^{2}\right)=x^{2} \otimes 1+(1+\beta) x g \otimes x+g^{2} \otimes x^{2}$. Let $x^{2}=\alpha 1+\gamma x$. Then applying $\varepsilon$, we see that $\alpha=0$ and $x^{2}=\gamma x$ so that $\Delta\left(x^{2}\right)=\gamma x \otimes 1+\gamma g \otimes x$. Thus $\beta=-1$ and $\gamma g \otimes x=g^{2} \otimes \gamma x$ so that $\gamma=0$. Since $\beta=-1,2 \mid m$ and the subalgebra generated by $g$ and $x$ is a pointed sub-Hopf algebra of $H$ of dimension $2 m=4 n$ for some $n \geq 1$.

2.8. Hopf algebras of dimension $4 p$. This section contains a brief overview of what is known for dimension $4 p$. Knowledge of the classification in this dimension is of course necessary to understand dimension $8 p$ which we study in the last section of this note. Recall that for dimension 12 the classification is due to [AN1], F], [N5].

Up to isomorphism, the semisimple Hopf algebras of dimension $4 p$ consist of group algebras and their duals, and also of two self-dual Hopf algebras, constructed by Gelaki in Gel1, which we will denote by $\mathcal{G}_{1}$ and $\mathcal{G}_{2}$. Both have group of grouplikes of order 4 with $G\left(\mathcal{G}_{1}\right) \cong C_{4}$ and $G\left(\mathcal{G}_{2}\right) \cong C_{2} \times C_{2}$.

2.8.1. Nonsemisimple pointed Hopf algebras of dimension 4p. All pointed Hopf algebras of dimension $4 p$ have group of grouplikes isomorphic to $C_{2 p}$ and are described in [AN1, A.1].

In particular, let $\mathcal{A}$ be a pointed Hopf algebra of dimension $4 p$. Then, with $g$ denoting a generator of $C_{2 p}$, and $\xi$ a primitive $p$ th root of unity, $\mathcal{A}$ is isomorphic to exactly one of the following.

$$
\begin{aligned}
& \mathcal{A}(-1,0):=\mathbb{k}\left\langle g, x \mid g^{2 p}-1=x^{2}=g x+x g=0\right\rangle, \\
& \Delta(g)=g \otimes g, \quad \Delta(x)=x \otimes 1+g \otimes x . \\
& \mathcal{A}(-1,0)^{*}:=\mathbb{k}\left\langle g, x \mid g^{2 p}-1=x^{2}=g x+\xi x g=0\right\rangle, \\
& \Delta(g)=g \otimes g, \quad \Delta(x)=x \otimes 1+g^{p} \otimes x . \\
& \mathcal{A}(-1,1):=\mathbb{k}\left\langle g, x \mid g^{2 p}-1=x^{2}-g^{2}+1=g x+x g=0\right\rangle, \\
& \Delta(g)=g \otimes g, \quad \Delta(x)=x \otimes 1+g \otimes x . \\
& H_{4} \otimes \mathbb{k} C_{p}:=\mathbb{k}\left\langle g, x \mid g^{2 p}-1=x^{2}=g x+x g=0\right\rangle, \\
& \Delta(g)=g \otimes g, \quad \Delta(x)=x \otimes 1+g^{p} \otimes x .
\end{aligned}
$$

Note that $H_{4} \otimes \mathbb{k} C_{p}$ is self-dual. The Hopf algebra $\mathcal{A}(-1,1)$ is a nontrivial lifting of $\mathcal{A}(-1,0)$ and has nonpointed dual. The nonpointed Hopf algebra $\mathcal{A}(-1,1)^{*}$ contains a copy of the Sweedler Hopf algebra and as a coalgebra $\mathcal{A}(-1,1)^{*} \cong H_{4} \otimes \mathcal{M}^{*}(2, \mathbb{k})^{p-1}$. The Hopf algebras $\mathcal{A}(-1,0)$ and $\mathcal{A}(-1,1)$ do not have sub-Hopf algebras isomorphic to $H_{4}$ but $\mathcal{A}(-1,0)^{*}$ and $H_{4} \otimes \mathbb{k} C_{p}$ do. In all four cases, $S^{4}=$ id. In Section 4 we will use this notation for these pointed Hopf algebras. 
2.8.2. Nonsemisimple nonpointed Hopf algebras of dimension $4 p$. These Hopf algebras were studied in $\mathrm{ChNg}$ with the classification completed for $p=3,5,7,11$. The main theorems of $\mathrm{ChNg}$ are:

Theorem 2.18. ChNg, Theorem I] For $H$ a nonsemisimple Hopf algebra of dimension $4 p$, then $H$ is pointed if and only if $|G(H)|>2$.

Theorem 2.19. ChNg, Theorem II] For $H$ a nonsemisimple Hopf algebra of dimension $4 p$ where $p \leq 11$ is an odd prime, then $H$ or $H^{*}$ is pointed.

2.8.3. Applications of counting arguments. We end this section by applying the preceding preliminary material to give some simple alternate arguments for known facts about nonsemisimple nonpointed Hopf algebras of dimension $4 p$.

Proposition 2.20. Let $H$ be a nonsemisimple nonpointed Hopf algebra of dimension $4 p$. Then $|G(H)| \notin\{p, 2 p\}$.

Proof. Suppose that $|G(H)|=p$ and then $H_{0}=\mathbb{k} G(H) \oplus\left(\oplus_{i=1}^{t} D_{i}^{n_{i}}\right)$ where the $D_{i}$ are simple subcoalgebras of dimension $d_{i}^{2}$ with $1<d_{1}<d_{2}<\ldots<d_{t}$. If $p$ divides some $d_{i}$, then $\operatorname{dim} H_{0} \geq p+p^{2}=$ $p(1+p) \geq 4 p$ since $p \geq 3$, a contradiction. Thus $\left(p, d_{i}\right)=1$ for all $i$ and $p$ must divide $n_{i}$ by Lemma 2.7. Then $\operatorname{dim} H_{0} \geq p+4 p>4 p$, a contradiction. An analogous proof shows that $|G(H)| \neq 2 p$.

Proposition 2.21. Suppose that $H$ is generated as an algebra by a simple coalgebra $D$ of dimension 4 which is stable by the antipode. Then $H^{*}$ is pointed.

Proof. By Proposition 2.13, $H$ fits into a central exact sequence of Hopf algebras

$$
\mathbb{K}^{G} \hookrightarrow H \rightarrow A,
$$

with $A$ nonsemisimple and $A^{*}$ pointed. Since $\operatorname{dim} A$ divides $4 p$, then $\operatorname{dim} A \in\{1,2,4, p, 2 p, 4 p\}$. We will show that $\operatorname{dim} A=4 p$ so that $H \cong A$ and thus $H^{*}$ is pointed.

Since $H$ is nonsemisimple, $\operatorname{dim} A>1$. If $\operatorname{dim} A=2, p$ or $2 p$, then $A$ is semisimple by the classification of Hopf algebras of these dimensions, see [S], [W], [Z], Ng3. Since $\mathbb{k}^{G}$ is semisimple, this would imply that $H$ is also semisimple, a contradiction.

If $\operatorname{dim} A=4$, then $\operatorname{dim} \mathbb{k}^{G}=p$ and this implies that $\mathbb{k}^{G} \simeq \mathbb{k} C_{p}$. Thus $C_{p} \subseteq G(H)$ so that $|G(H)|=p$ or $2 p$, a contradiction by Proposition 2.20, Thus $A=H$ and $H^{*}$ is pointed.

\section{HOPF ALGEBRAS OF DIMENSION rpq}

In this section, $H$ will be a Hopf algebra of dimension $r p q$, with $r<p<q$ primes. Recently, Etingof, Nikshych and Ostrik [ENO, finished the classification of the semisimple Hopf algebras of dimension $r p q$ and $r p^{2}$. Specifically, they prove that all semisimple Hopf algebras of these dimensions can be obtained as abelian extensions (Kac Algebras). Then, the classification follows by a result of Natale [N1]. Thus, we will assume that $H$ is nonsemisimple. One purpose of this section is to apply counting arguments in the style of D. Fukuda as we did in [BG].

Remark 3.1. Recall that by Lemma 2.8 a nonsemisimple Hopf algebra $H$ of dimension $r p q$ is nonpointed, has no pointed sub-Hopf algebras and has no pointed quotient Hopf algebras. In particular, $H$ cannot be generated by a simple 4-dimensional subcoalgebra $C$ stable under the antipode. For then by Proposition 2.13, $H \cong \mathbb{k}^{G}$, which is semisimple, a contradiction.

Also $H$ cannot have the Chevalley property. The proof is based on the proof of [AN1, Lemma A.2].

Proposition 3.2. No nonsemisimple Hopf algebra $H$ of dimension rpq has the Chevalley property. 
Proof. Suppose that $H$ has the Chevalley property. Then $\operatorname{dim} H_{0} \mid \operatorname{dim} H$ and since $H$ is not pointed or cosemisimple, $1<\operatorname{dim} H_{0}<\operatorname{dim} H$. Then $\operatorname{dim} H_{0}=s t$, where $s, t \in\{r, p, q\}$ and $s<t$. But by [EGel1], [So, [N1] or if $s=2$ by [Ng3, all semisimple Hopf algebras of dimension st are trivial, i.e. isomorphic to a group algebra or the dual of a group algebra. Hence $H_{0} \simeq \mathbb{k}^{F}$ with $F$ a nonabelian group of order st; in particular, $s \mid(t-1)$. Consider now the coradical filtration on $H$ and the associated graded Hopf algebra gr $H$. Then write gr $H \simeq R \# \mathbb{k}^{F}$ with $R$ the diagram of $H$. Then $(\operatorname{gr} H)^{*} \simeq R^{*} \# \mathbb{k} F$, which implies that $(\operatorname{gr} H)^{*}$ is pointed. This cannot occur, since $\operatorname{dim}(\operatorname{gr} H)^{*}=$ $\operatorname{dim} \operatorname{gr} H=\operatorname{dim} H=r p q$. Hence, $H_{0}$ cannot be a sub-Hopf algebra.

We note that all Hopf algebras of dimension $30=2 \cdot 3 \cdot 5$ are group algebras or duals of group algebras by [Fu3] but the classification of the other Hopf algebras of dimension $r p q$ with $r p q<100$, namely dimensions $42,66,70$ and 78 is still open. We make a few observations about these cases.

Remark 3.3. (i) A nonsemisimple Hopf algebra of dimension $2 p q$ cannot have a semisimple sub-Hopf algebra $A$ of dimension $p q$. For if this were the case, there would be a Hopf algebra epimorphism $\pi: H^{*} \rightarrow A^{*}$ and we apply Proposition 2.17. Since $H^{*}$ has no nontrivial skew-primitive elements, $\left(H^{*}\right)^{c o \pi}=\mathbb{k} C_{2}$ and we have an exact sequence of Hopf algebras $\mathbb{k} C_{2} \hookrightarrow H^{*} \rightarrow A^{*}$. Thus if $A$, and thus $A^{*}$, were semisimple, $H^{*}$ and $H$ would be also.

(ii) Suppose that $H$ is nonsemisimple of dimension $2 p q$ where all Hopf algebras of dimension $p q$ are semisimple. Then by (i) above, $H$ has no sub-Hopf algebras of dimension $p q$. Suppose that $|G(H)|>2$ and let $C$ be a simple subcoalgebra of dimension greater than 1 . We will show that $C$ generates $H$.

Indeed, let $\mathcal{C}:=\langle C\rangle$ be the sub-Hopf algebra generated by $C$. Then $\operatorname{dim} \mathcal{C} \in\{2 p, 2 q, 2 p q\}$. If $\mathcal{C} \neq H$, then $\mathcal{C} \cong \mathbb{k}^{\mathbb{D}_{m}}$ with $m \in\{p, q\}$. Then $H$ is generated by $\mathcal{C}$ and $\mathbb{k} G(H)$, so by $\operatorname{Remark} 2.1, H$ is semisimple, a contradiction.

Lemma 3.4. Suppose that $H$ has dimension $2 p q$ with $2<p<q$. Then

(i) $|G(H)| \neq p q$.

(ii) If $p \leq 7$ then $|G(H)| \neq 2 q$ and if $q \leq 7$ then $|G(H)| \neq 2 p$.

(iii) If $p \leq 5$ then $|G(H)| \neq q$.

Proof. (i) The statement was proved in Remark 3.3(i).

(ii) If $|G(H)|=2 p$, since for all $d$, by Lemma 2.7, $2 p$ divides $\operatorname{dim} H_{0, d}=n d^{2}$ for some $n \geq 1$ then $\operatorname{dim} H_{0} \geq 2 p+4 p=6 p$. Then by Proposition 2.9)(ii) and Lemma 2.7, $\operatorname{dim} H \geq 6 p+2 p(5)+4 p=20 p$, a contradiction if $q \leq 7$.

If $|G|=2 q$ and $p \leq 7$ the argument is the same.

(iii) Assume $|G(H)|=q$. For $G, \mathcal{D}$ as above, $\operatorname{dim} H_{0} \geq q+4 q=5 q, 2 \operatorname{dim} P_{1}^{G, \mathcal{D}} \geq 4 q, \operatorname{dim} P^{G, G}$ and $\operatorname{dim} P^{\mathcal{D}, \mathcal{D}}$ must be divisible by $q$ and so $\operatorname{dim} H \geq 11 q>2(5) q$, a contradiction.

Corollary 3.5. (i) If $\operatorname{dim} H=42$, then $|G(H)| \notin\{21,14,7,6\}$.

(ii) If $\operatorname{dim} H=70$, then $|G(H)| \notin\{35,14,10,7\}$.

(iii) If $\operatorname{dim} H=66$, then $|G(H)| \notin\{33,22,11\}$.

(iv) If $\operatorname{dim} H=78$, then $|G(H)| \notin\{39,26,13\}$.

Next we show that for Hopf algebras of dimension $66, G(H)$ does not have order 6 .

Lemma 3.6. If $\operatorname{dim} H=6 p$ with $p<13$, then $|G(H)| \neq 6$.

Proof. First we suppose that $H$ has a simple subcoalgebra of dimension 4 and consider various cases. Let $G:=G(H)$, the group of grouplikes of $H$ of order 6 , and let $\mathcal{D}$ denote the set of simple subcoalgebras of dimension 4 . 
(i) Suppose that $H_{0}=\mathbb{k} G \oplus \mathcal{M}^{*}(2, \mathbb{k})^{3}$ so that $\operatorname{dim} H_{0}=18$. Since, by Remarks 3.1 and 3.3 , no $D \in \mathcal{D}$ is stable by the antipode, then no $D \in \mathcal{D}$ can be fixed by $S^{2}$ either. Thus if $P_{1}^{1, D}$ is nondegenerate, so are $P_{1}^{1, S^{2}(D)}, P_{1}^{S(D), 1}$ and $P_{1}^{S^{3}(D)=D, 1}$ and $2 \operatorname{dim} P_{1}^{G, \mathcal{D}} \geq 2(6) 4=48$. Since $P^{G, G}$ has nonzero dimension divisible by 6 and $P^{\mathcal{D}, \mathcal{D}}$ has nonzero dimension divisible by 12 , then the dimension of $H$ is at least $18+48+6+12=84$, a contradiction.

(ii) Suppose that $H_{0}=\mathbb{k} G \oplus \mathcal{M}^{*}(2, \mathbb{k})^{3 t}$ with $t \geq 2$ so that $\operatorname{dim} H_{0}=6+4(3 t) \geq 30$. Since for some integers $\ell, m, n \geq 1,2 \operatorname{dim} P^{G, \mathcal{D}}=24 \ell, \operatorname{dim} P^{G, G}=6 m$, $\operatorname{dim} P^{\mathcal{D}, \mathcal{D}}=12 n$, then $\operatorname{dim} H \geq 72$, so that we obtain a contradiction if $p<13$.

(iii) Suppose that $H_{0}=\mathbb{k} G \oplus \mathcal{M}^{*}(2, \mathbb{k})^{3 t} \oplus E_{1} \ldots \oplus E_{N}$ where $t, N \geq 1$ and the $E_{i}$ are simple subcoalgebras of dimension greater than 4 . Let $\mathcal{E}$ denote the set of $E_{i}$ and $\mathcal{D}$ the set of simple subcoalgebras of dimension 4 . Then $\operatorname{dim} H_{0} \geq 6+12+18=36$. If $P^{G, \mathcal{E}} \neq 0$, then $2 \operatorname{dim} P^{G, \mathcal{E}} \geq$ $2(6)(3)=36, \operatorname{dim} P^{\mathcal{E}, \mathcal{E}} \geq 9$ and so $\operatorname{dim} H \geq 81$, contradiction. Thus $P^{G, \mathcal{D}} \neq 0$. If $t=1$, then as in (i) above $2 \operatorname{dim} P_{1}^{G, \mathcal{D}} \geq 2(6) 4=48$, so that $\operatorname{dim} H \geq 36+48=84$, a contradiction. If $t \geq 2$, then $\operatorname{dim} H_{0} \geq 48$ and $2 \operatorname{dim} P_{1}^{G, \mathcal{D}} \geq 24$ so that $\operatorname{dim} H_{1} \geq 72$. But $P_{2}^{\mathcal{D}, \mathcal{D}}, P_{2}^{G, G}$ are nondegenerate, so that $\operatorname{dim} H_{2} \geq 80$, a contradiction.

Now suppose that $H$ has no simple subcoalgebras of dimension 4 and $H_{0}=\mathbb{k} G \oplus E_{1} \ldots \oplus E_{t}$ where the $E_{i}$ are simple subcoalgebras of dimension at least 9 so that $\operatorname{dim} H_{0} \geq 6+18=24$. Let $\mathcal{E}$ denote the set of simple coalgebras $E_{i}$. Then $2 \operatorname{dim} P_{1}^{G, \mathcal{E}} \geq 2(6)(3)=36, \operatorname{dim} P^{G, G} \geq 6, \operatorname{dim} P^{\mathcal{E}, \mathcal{E}} \geq 9$ and must be divisible by 6 so that $\operatorname{dim} P^{\mathcal{E}, \mathcal{E}} \geq 12$. But also $\operatorname{dim} P^{\mathcal{E}, \mathcal{E}}$ must be a sum of squares larger than 4 so that $\operatorname{dim} P^{\mathcal{E}, \mathcal{E}}>12$. Thus $\operatorname{dim} H>24+36+6+12=78$, a contradiction.

Note that in the proof above, the only place where $p \neq 13$ was used was in case (ii). There if $p=13$ we must have that $\ell=n=1$ and $m=2$.

Next we show that for dimension 70 the group of grouplikes must have order 1 or 2 .

Lemma 3.7. If $\operatorname{dim} H=70$ then $G(H) ¥ C_{5}$.

Proof. Again, we suppose first that $H$ has a simple subcoalgebra of dimension 4 and consider various cases. Let $\mathcal{D}$ denote the set of simple subcoalgebras of dimension 4 and let $G:=G(H) \cong C_{5}$.

(i) Suppose that $H_{0}=\mathbb{k} C_{5} \oplus D_{1} \oplus \ldots \oplus D_{5}$ where $D_{i} \cong \mathcal{M}^{*}(2, \mathbb{k})$. Since no $D_{i}$ is stable under the antipode, we may assume that $S\left(D_{i}\right)=D_{i+1}$, subscripts modulo 5. For if $S^{2}\left(D_{1}\right)=D_{1}$, then $S$ would permute $D_{3}, D_{4}, D_{5}$. But by Corollary 3.5, $\left|G\left(H^{*}\right)\right| \in\{1,2,5\}$ and so 3 does not divide the order of $S$. Thus by Proposition 2.9(i), $P_{1}^{1, D_{i}}$ is nondegenerate for all $i$ and $2 \operatorname{dim} P^{G, \mathcal{D}} \geq 2(5)(10)=100$, a contradiction.

(ii) Suppose that $H_{0}=\mathbb{k} C_{5} \oplus \mathcal{M}^{*}(\mathbb{k}, 2)^{5 t}$ where $t>1$. Then $\operatorname{dim} H_{0} \geq 5+10(4)=45,2 \operatorname{dim} P^{G, \mathcal{D}} \geq$ $2(5)(2)=20, \operatorname{dim} P^{G, G} \geq 5$, and $\operatorname{dim} P^{\mathcal{D}, \mathcal{D}} \geq 4$, so that $\operatorname{dim} H \geq 74$.

(iii) Let $H_{0}=\mathbb{k}_{5} \oplus \mathcal{M}^{*}(2, \mathbb{k})^{5 t} \oplus E$, where $t \geq 1$ and $0 \neq E$ is a sum of simple subcoalgebras $E_{i}$ of dimension greater than 4 . Let $\mathcal{E}$ denote the set of $E_{i}$. If the dimensions of any of the $E_{i}$ are relatively prime to 5 , then $\operatorname{dim} H_{0} \geq 5+20+5(9)=70$, a contradiction. The only remaining case is $H_{0}=\mathbb{k} C_{5} \oplus \mathcal{M}^{*}(2, \mathbb{k})^{5} \oplus \mathcal{M}^{*}(5, \mathbb{k})$; here $t=1$ or else $H=H_{0}$. Then $\operatorname{dim} H_{0}=50,2 \operatorname{dim} P_{1}^{G, \mathcal{D}} \geq$ $2(5)(2)=20$ and this is a contradiction since $H \neq H_{1}$.

Thus $H$ cannot have a simple subcoalgebra of dimension 4 . The only other possibilities for $H_{0}$ are $H_{0}=\mathbb{k} C_{5} \oplus \mathcal{M}^{*}(3, \mathbb{k})^{5}$ and $H_{0}=\mathbb{k} C_{5} \oplus \mathcal{M}^{*}(5, \mathbb{k})^{t}$ with $t=1,2$. In the first case, $\operatorname{dim} H_{0}=50$, and for $\mathcal{E}$ the set of simple subcoalgebras of dimension $9,2 \operatorname{dim} P^{G, \mathcal{E}} \geq 2(5)(3)=30$, a contradiction. In the second case, first let $t=1$ and here let $\mathcal{E}$ be the set of simple subcoalgebras of dimension 25 . Then $\operatorname{dim} H_{0}=30$ and $2 \operatorname{dim} P^{G, \mathcal{E}} \geq 2(5)(5)=50$, a contradiction. The proof for $t=2$ is the same. 
Corollary 3.8. If $H$ is a nonsemisimple Hopf algebra of dimension 70 , then $|G(H)|=1,2$.

Remark 3.9. (i) To summarize, we have that for $H$ of dimension 42,66, $|G(H)| \in\{1,2,3\}$, for $H$ of dimension $70,|G(H)| \in\{1,2\}$ and for $H$ of dimension $78,|G(H)| \in\{1,2,3,6\}$.

(ii) If $\operatorname{dim} H=42$ and $G(H) \cong C_{3}$, then dimension arguments such as those above show that $H$ has following form: $H_{0} \cong \mathbb{k} C_{3} \oplus C$ with $C \cong \mathcal{M}^{*}(3, \mathbb{k}), 2 \operatorname{dim} P^{G, C}=18, \operatorname{dim} P^{G, G}=3, \operatorname{dim} P^{C, C}=9$.

\section{Hopf algebras of Dimension $8 p$}

In this section we prove some results for Hopf algebras of dimension $8 p$.

4.1. Hopf algebras of dimension 8. The structure of Hopf algebras of dimension 8 or dimension $4 p$ naturally plays a role in the classification of Hopf algebras of dimension $8 p$. Hopf algebras of dimension $4 p$ were discussed in Section 2.8.1. including a complete description of the pointed ones.

For dimension 8 the semisimple Hopf algebras are group algebras, duals of group algebras or the noncommutative noncocommutative semisimple Hopf algebra of dimension 8, denoted by $A_{8}$ Mas2. This Hopf algebra is self-dual and $G\left(A_{8}\right) \cong C_{2} \times C_{2}$; it is constructed as an extension of $\mathbb{k}\left[C_{2} \times C_{2}\right]$ by $\mathbb{k} C_{2}$. All other Hopf algebras of dimension 8 are pointed or basic.

Let $\xi$ be a primitive $4^{\text {th }}$ root of 1 . By [S], every pointed nonsemisimple Hopf algebra of dimension 8 is isomorphic to exactly one of the Hopf algebras listed below:

$$
\begin{gathered}
\mathcal{A}_{2}:=\mathbb{k}\left\langle g, x, y \mid g^{2}-1=x^{2}=y^{2}=g x+x g=g y+y g=x y+y x=0\right\rangle, \\
\Delta(g)=g \otimes g, \quad \Delta(x)=x \otimes 1+g \otimes x, \quad \Delta(y)=y \otimes 1+g \otimes y . \\
\mathcal{A}_{4}^{\prime}:=\mathbb{k}\left\langle g, x \mid g^{4}-1=x^{2}=g x+x g=0\right\rangle, \\
\Delta(g)=g \otimes g, \quad \Delta(x)=x \otimes 1+g \otimes x ; \\
\mathcal{A}_{4}^{\prime \prime}:=\mathbb{k}\left\langle g, x \mid g^{4}-1=x^{2}-g^{2}+1=g x+x g=0\right\rangle, \\
\Delta(g)=g \otimes g, \quad \Delta(x)=x \otimes 1+g \otimes x ; \\
\mathcal{A}_{4, \xi}^{\prime \prime \prime}:=\mathbb{k}\left\langle g, x \mid g^{4}-1=x^{2}=g x-\xi x g=0\right\rangle, \\
\Delta(g)=g \otimes g, \quad \Delta(x)=x \otimes 1+g^{2} \otimes x ; \\
\mathcal{A}_{2,2}:=\mathbb{k}\left\langle g, h, x \mid g^{2}=h^{2}=1, x^{2}=g x+x g=h x+x h=g h-h g=0\right\rangle, \\
\Delta(g)=g \otimes g, \quad \Delta(h)=h \otimes h, \quad \Delta(x)=x \otimes 1+g \otimes x .
\end{gathered}
$$

Except for $\mathcal{A}_{4}^{\prime \prime}$, these pointed Hopf algebras have pointed duals. We have the following isomorphisms: $\mathcal{A}_{2} \simeq\left(\mathcal{A}_{2}\right)^{*}, \mathcal{A}_{4, \xi}^{\prime \prime \prime} \simeq \mathcal{A}_{4,-\xi}^{\prime \prime \prime} \simeq\left(\mathcal{A}_{4}^{\prime}\right)^{*}$ and $\mathcal{A}_{2,2} \simeq\left(\mathcal{A}_{2,2}\right)^{*}[\mathrm{~S}]$. Moreover, one can check case by case that $\mathcal{A}_{2}, \mathcal{A}_{4, \xi}^{\prime \prime \prime}$ and $\mathcal{A}_{2,2}$ have sub-Hopf algebras isomorphic to $H_{4}$ and $\mathcal{A}_{4}^{\prime}, \mathcal{A}_{4}^{\prime \prime}$ do not.

Let $\mathcal{K}=\left(\mathcal{A}_{4}^{\prime \prime}\right)^{*}$. Up to isomorphism $\mathcal{K}$ is the only Hopf algebra of dimension 8 which is neither semisimple nor pointed. The next remark is essentially [GV, Lemma 3.3].

Remark 4.1. (i) $\mathcal{K}$ is generated as an algebra by the elements $a, b, c, d$ satisfying the relations

$$
\begin{array}{lllll}
a b=\xi b a & a c=\xi c a & 0=c b=b c & c d=\xi d c & b d=\xi d b \\
a d=d a & a d=1 & 0=b^{2}=c^{2} & a^{2} c=b & a^{4}=1
\end{array}
$$

(ii) The elements $a=e_{11}, b=e_{12}, c=e_{21}, d=e_{22}$ form a matrix-like basis and

$$
\Delta\left(a^{2}\right)=a^{2} \otimes a^{2} \text { and } \Delta(a c)=a c \otimes a^{2}+1 \otimes a c .
$$

(iii) $\mathcal{K} \simeq H_{4} \oplus \mathcal{M}^{*}(2, \mathbb{k})$ as coalgebras.

Using Remark 4.1, one sees that $\mathcal{K}$ is a finite dimensional quotient of the quantum group $\mathcal{O}_{\xi}\left(S L_{2}\right)$; this is consistent with Proposition 2.13 , 
4.2. Nonsemisimple Hopf algebras of dimension $8 p$. Throughout this section, unless otherwise stated, we will assume that $H$ is a nonsemisimple nonpointed nonbasic Hopf algebra of dimension $8 p$. Also recall that $p$ denotes an odd prime. Our strategy will be to study the possible orders for the grouplikes in $H$ where $\operatorname{dim} H=8 p$. In this section we prove Theorem $\mathrm{A}$.

4.2.1. Group of grouplikes divisible by $p$. In this subsection we concentrate on general results for Hopf algebras of dimension $8 p$ with $|G(H)|$ divisible by $p$.

Proposition 4.2. $|G(H)| \neq 8 p, 4 p$ or $p$.

Proof. For $H$ non-cosemisimple, $|G(H)| \neq 8 p$. If $|G(H)|=4 p$, since $H$ is not pointed, $H_{0}=\mathbb{k} G(H) \oplus E$ with $E$ the sum of simple coalgebras of dimension bigger than 1 . Since $4 p$ must divide $\operatorname{dim}(E)$ by Lemma 2.7, then we must have $H=H_{0}$, impossible because $H$ is not cosemisimple.

If $|G(H)|=p$, then $H$ has no nontrivial skew-primitives by Lemma 2.8. Now we use counting arguments as in the previous sections. Suppose that $H_{0}=\mathbb{k} G(H) \oplus D_{1}^{s_{1}} \oplus \ldots D_{t}^{s_{t}}$ for $D_{i}$ simple of dimension $n_{i}^{2}$ and $2 \leq n_{1}<\ldots<n_{t}$. Let $\mathcal{D}$ denote the set of simple coalgebras $D_{i}$. Then by Proposition 2.9(i) and Lemma 2.6(ii), $2 \operatorname{dim} P^{C_{p}, \mathcal{D}} \geq 2 p n_{1}$. If $p \operatorname{divides} n_{1}$, then $\operatorname{dim} H \geq \operatorname{dim} H_{0}+$ $2 \operatorname{dim} P^{C_{p}, \mathcal{D}} \geq p+p^{2}+2 p^{2}=p(1+3 p)>8 p$ since $p \geq 3$. If $\left(p, n_{1}\right)=1$, then $p$ divides $s_{1}$ and $\operatorname{dim} H \geq p+4 p+4 p>8 p$. Thus in each case, we arrive at a contradiction.

Thus, if $|G(H)|=2 p$, then $H$ cannot have the Chevalley property. For, suppose that $H_{0}$ is a sub-Hopf algebra of $H$. Since $H$ is not pointed or cosemisimple, dim $H_{0}=4 p$. Since the semisimple Hopf algebras $\mathcal{G}_{i}$ have grouplikes of order 4 , then $H_{0} \cong \mathbb{k}^{\Gamma}$ where $\Gamma$ is a nonabelian group of order $4 p$. But then $H_{0}=\mathbb{k}^{\Gamma} \cong \mathbb{k} G\left(H_{0}\right) \oplus D$ where $\left|G\left(H_{0}\right)\right|=2 p$ and $D$ is a sum of simple coalgebras. By Lemma 2.7. $D$ is a sum of matrix coalgebras of dimension $d>1$ and $2 p=n d^{2}$ which is impossible.

Remark 4.3. As in the proof above, we use Lemma 2.8 together with counting arguments to eliminate the possibility that $|G(H)|=8$ for some small dimensions. Let $\operatorname{dim} H=8 p$ with $p \in\{3,5,7\}$ and suppose $|G(H)|=8$. By Lemma 2.8, $H$ has no nontrivial skew primitive elements. Since $\operatorname{dim} H_{0}=$ $8+8 m$ for some integer $m \geq 1$, by Lemma 2.9(ii), we have that $\operatorname{dim} H \geq 16+40+4=60$.

The next proposition shows that type $(8,2 p)$ is impossible.

Proposition 4.4. If $|G(H)|=2 p$ then $H^{*}$ has no semisimple sub-Hopf algebra $L$ of dimension 8.

Proof. Suppose $H^{*}$ contains a semisimple sub-Hopf algebra $L$ of dimension 8 and let $\Gamma$ be a subgroup of $G(H)$ of order $p$. Since $L^{*}$ is semisimple and has no grouplike elements of order $p, \mathbb{k} \Gamma \hookrightarrow H \rightarrow L^{*}$ is an exact sequence of Hopf algebras. This implies that $H$ is semisimple, a contradiction.

The next proposition determines the coalgebra structure of $H$ when $|G(H)|=2 p$.

Proposition 4.5. Suppose $|G(H)|=2 p$.

(i) $H$ contains a pointed sub-Hopf algebra $\mathcal{A}$ of dimension $4 p$ and as a coalgebra $H \cong \mathcal{A} \oplus$ $\mathcal{M}^{*}(2, \mathbb{k})^{p}$.

(ii) If $H^{*}$ is generated by a simple subcoalgebra of dimension 4 fixed by $S_{H^{*}}^{4}$ then $S_{H}$ has order 4 .

Proof. (i) Since $H$ is not pointed, $H_{0}=\mathbb{k} G(H) \oplus D_{1} \oplus \ldots \oplus D_{t}$ where the $D_{i}$ are simple coalgebras of dimension greater than 1 . Suppose that $H_{0, m p} \neq 0$ where $m \geq 1$. Then $\operatorname{dim}\left(H_{0, m p}\right) \geq 2 p^{2}$ and thus $\operatorname{dim}\left(H_{0}\right) \geq 2 p+2 p^{2}=p(2+2 p) \geq 8 p$ since $p \geq 3$, and this is impossible since $H$ is nonsemisimple. If $H_{o, d} \neq 0$ for $(d, p)=1$ and $d>2$ then $\operatorname{dim}\left(H_{0}\right) \geq 2 p+p d^{2}=p\left(2+d^{2}\right)>8 p$ which is also impossible. Thus $D_{i}=\mathcal{M}^{*}(2, \mathbb{k})$ for all $i$, and $H_{0} \cong \mathbb{k} G(H) \oplus \mathcal{M}^{*}(2, \mathbb{k})^{p}$ as coalgebras. 
By Proposition 2.9(ii), $H$ has a nontrivial skew-primitive $x$ and $x$ together with $G(H)$ generates a pointed sub-Hopf algebra $\mathcal{A}$ of $H$ of dimension $4 p$ and (i) is proved.

(ii) By (i) there is a Hopf algebra projection $\pi: H^{*} \rightarrow \mathcal{A}^{*}$ for $\mathcal{A}$ the pointed Hopf algebra of dimension $4 p$ from (i). Then $S_{\mathcal{A}}$ and $S_{\mathcal{A}^{*}}$ have order 4 . Suppose $D \cong \mathcal{M}^{*}(2, \mathbb{k}) \subset H^{*}$ is stable under $S_{H^{*}}^{4}$ and generates $H^{*}$, and suppose that $S_{H^{*}}^{4}$ has order $N>1$. Let e be a multiplicative matrix for $D$ as in Theorem 2.10 such that $S_{H^{*}}^{4}\left(e_{i j}\right)=\omega^{i-j} e_{i j}$ where $\omega$ is a primitive $N^{\text {th }}$ root of unity. Then if $i \neq j, \pi\left(e_{i j}\right)=0$ and thus $\operatorname{dim} \pi(D)<3$. By Theorem 2.11, $\pi(D) \subseteq G\left(\mathcal{A}^{*}\right)$ so that $\pi(D)$ does not generate $\mathcal{A}^{*}$, contradicting the fact that $D$ generates $H^{*}$.

Next we show that if $|G(H)|=2 p$, then $H^{*}$ cannot contain a copy of the Sweedler Hopf algebra.

Proposition 4.6. Assume $|G(H)|=2 p$. Then $H^{*}$ has no sub-Hopf algebra isomorphic to $H_{4}$.

Proof. If $H^{*}$ contains a sub-Hopf algebra isomorphic to $H_{4}$, there exists a Hopf algebra epimorphism $\pi: H \rightarrow H_{4}$. Then, by Lemma 2.4, $\operatorname{dim} H^{\operatorname{co} \pi}=\operatorname{dim}{ }^{\operatorname{co} \pi} H=2 p$. Let $G(H)=\langle c\rangle \cong C_{2 p}$ and let $\Gamma=\left\langle c^{2}\right\rangle \cong C_{p}$. Since $p$ is odd, we have that $\mathbb{k} \Gamma$ is included both in $H^{\operatorname{co} \pi}$ and ${ }^{\operatorname{co} \pi} H$.

On the other hand, Proposition 4.5 implies that $H \simeq \mathcal{A} \oplus D$ where $D=D_{1} \oplus \cdots \oplus D_{p}$, with $D_{j} \simeq \mathcal{M}^{*}(2, \mathbb{k})$, for all $1 \leq j \leq p$. We will prove that for every $j, 1 \leq j \leq p, \operatorname{dim} D_{j}^{\operatorname{co} \pi} \geq 2$ or $\operatorname{dim}{ }^{\text {co } \pi} D_{j} \geq 2$. This fact leads to a contradiction. Indeed, suppose that for $n$ of the $D_{j}, \operatorname{dim} D_{j}^{\text {co } \pi} \geq 2$ and for the remaining $p-n$ coalgebras $D_{j}$, dim ${ }^{\text {co } \pi} D_{j} \geq 2$. Since $p$ is odd, either $2 n>p$ or $2(p-n)>p$ so that either $\operatorname{dim}^{c o \pi} D>p$ or $\operatorname{dim} D^{c o \pi}>p$. Since $\mathbb{k}\left\langle c^{2}\right\rangle$ lies in both the left and right coinvariants, this implies that either $\operatorname{dim}^{c o \pi} H>2 p$ or $\operatorname{dim} H^{c o \pi}>2 p$, and this gives the desired contradiction.

Fix a simple subcoalgebra $D_{j}$ and let $K=\left\langle D_{j}\right\rangle$, the sub-Hopf algebra of $H$ generated by $D_{j}$. Clearly, $\operatorname{dim} K=8,2 p, 4 p$ or $8 p$. We write $\pi$ also for $\left.\pi\right|_{K}$ when the meaning is clear. If $\pi$ maps $K$ onto $H_{4}$, then the result follows from Lemma 2.12 , in particular, $\operatorname{dim} K \neq 8 p$. If $\pi(K)=\mathbb{k}$, then $\left.\pi\right|_{K}=\varepsilon_{K}$. Hence for $\mathbf{d}=\left(d_{i j}\right)$ a multiplicative matrix for $D_{j}, \pi\left(d_{i j}\right)=\delta_{i j}$ and $D_{j}$ lies in both the left and right coinvariants. It remains to consider the case when $\pi(K)=\mathbb{k} G\left(H_{4}\right)=\mathbb{k}\langle g\rangle$ where $g$ generates $G\left(H_{4}\right) \simeq C_{2}$.

Assume $\operatorname{dim} K=8$. Since $K$ is nonpointed, by Subsection 4.1 we have that $K=\mathcal{K}=\left(\mathcal{A}_{4}^{\prime \prime}\right)^{*} \cong L \oplus D_{j}$ as coalgebras where $L \cong H_{4}$. Since $G(L) \subset G(H)$, we have that $c^{p} \in K$. Suppose $\pi\left(c^{p}\right)=1$. Since $\pi(c)$ is a grouplike element and $\left|G\left(H_{4}\right)\right|=2$, we have that $\pi(c)=1$. If $x$ is a nontrivial skew-primitive in $H_{4} \subset K$ such that $\pi(x)=0$, then $c, x$ lie in both ${ }^{c o \pi} H$ and $H^{c o \pi}$, contradicting the fact that the dimension of the coinvariants is $2 p$. Thus $\pi\left(c^{p}\right)=\pi(c)=g,{ }^{c o \pi} L=\{1, g x\}, L^{c o \pi}=\{1, x\}$. Since $\operatorname{dim}^{c o \pi} K=\operatorname{dim} K^{c o \pi}=4$, then we must have that $\operatorname{dim}^{c o \pi} D_{j}=\operatorname{dim} D_{j}^{c o \pi}=2$.

Next we will show that if $\operatorname{dim} \pi(K)=2$ then $K$ cannot have dimension $4 p$ or $2 p$. Suppose that $\operatorname{dim} K=4 p$. Then $\operatorname{dim} K^{c o \pi}=2 p=\operatorname{dim}^{c o \pi} K$ so that $K^{c o \pi}=H^{c o \pi}$ and the same for the left coinvariants. Thus $\mathbb{k}\left\langle c^{2}\right\rangle \cong \mathbb{k} C_{p} \subset K$. If $K$ is nonpointed semisimple, by the classification of semisimple Hopf algebras of dimension $4 p$ in Section 2.8, $p$ does not divide the order of $G(K)$ either if $K$ is the dual of a group algebra or if $K$ is one of the semisimple Hopf algebras in Gel1. If $K$ is not semisimple then by Theorem $2.18, K$ is pointed, a contradiction.

Finally, suppose now that $\operatorname{dim} K=2 p$ so that $K \cong \mathbb{K}^{\mathbb{D}^{p}}$ and $\operatorname{dim} K^{c o \pi}=p$. Let $\tilde{K}=\langle K, \Gamma\rangle$ be the sub-Hopf algebra of $H$ generated by $K$ and $\mathbb{k}\left\langle c^{2}\right\rangle$. Since $\tilde{K}$ is semisimple, then $\tilde{K} \neq H$ and so has dimension $4 p$. But $\tilde{K}$ is then a nonpointed semisimple sub-Hopf algebra of $H$ of dimension $4 p$ with a grouplike of order $p$, and this is impossible by the proof in the paragraph above.

The next proposition shows that type $(2 p, r)$ can occur only if $r=2,4$. 
Proposition 4.7. Suppose $|G(H)|=2 p$. Then

(i) $H$ fits into an exact sequence of Hopf algebras $\mathcal{A} \hookrightarrow H \rightarrow \mathbb{k} C_{2}$, where $\mathcal{A}$ is a pointed Hopf algebra of dimension $4 p$. In particular, $G(H)$ is cyclic.

(ii) If $\mathcal{A}^{*}$ is nonpointed, i.e., $\mathcal{A} \cong \mathcal{A}(-1,1)$, then $\operatorname{dim} H_{0}^{*}=8 p-4, G\left(H^{*}\right) \cong C_{4}$ and $H^{*}$ has a sub-Hopf algebra isomorphic to $\mathcal{A}_{4}^{\prime \prime}$.

(iii) If $\mathcal{A}^{*}$ is pointed then $\operatorname{dim} H_{0}^{*}=4 p$ and $\left|G\left(H^{*}\right)\right|$ is 2 or 4 . If $H^{*}$ has a nontrivial skew-primitive element, then $H^{*}$ has a sub-Hopf algebra isomorphic to $\mathcal{A}_{4}^{\prime \prime}$.

Proof. (i) Proposition 4.5 implies that $H \simeq \mathcal{A} \oplus \mathcal{M}^{*}(2, k)^{p}$, with $\mathcal{A}$ a pointed Hopf algebra of dimension $4 p$. Dualizing this inclusion we get a Hopf algebra epimorphism $\pi: H^{*} \rightarrow \mathcal{A}^{*}$ and $\operatorname{dim} H^{*}=2 \operatorname{dim} \mathcal{A}^{*}$. Thus by Proposition 2.17, $R:=\left(H^{*}\right)^{c o \pi}=\mathbb{k}\{1, x\}$ with $x$ a (possibly trivial) $(1, g)$-primitive element for some grouplike $g \in G\left(H^{*}\right)$ with ord $g=2 n, n \geq 1$. Since $\operatorname{dim} H^{*}=8 p$, we have that ord $g=2,4$ or $2 p$.

Assume ord $g=2$. If $x$ is a nontrivial skew-primitive, then by Proposition 2.17 $H^{*}$ has a sub-Hopf algebra isomorphic to $H_{4}$ and this is impossible by Proposition 4.6. Thus $x \in \mathbb{k} G\left(H^{*}\right)$ and $R$ is a Hopf algebra isomorphic to the group algebra $\mathbb{k} C_{2}$. In particular, $H$ fits into the exact sequence of Hopf algebras $\mathcal{A} \hookrightarrow H \rightarrow \mathbb{k} C_{2}$.

We show now that the other cases are not possible. Assume ord $g=4$. Then by Proposition 2.17, $x$ must be nontrivial and $H^{*}$ contains a pointed sub-Hopf algebra $L$ of dimension 8 . By inspection on the pointed Hopf algebras of dimension 8 in Section 4.1, we must have that $L \simeq \mathcal{A}_{4}^{\prime}$ and consequently $L^{*}$ is pointed. This implies that $\mathbb{k} C_{p} \hookrightarrow H \rightarrow L^{*}$ is an exact sequence of Hopf algebras and by $\mathrm{G}$, Thm. 2.1] $H$ would be pointed, a contradiction.

Finally assume that ord $g=2 p$. Then $G\left(H^{*}\right) \cong C_{2 p}$ and by Proposition 4.5)(i), as coalgebras $H^{*} \simeq \mathcal{B} \oplus \mathcal{M}^{*}(2, \mathbb{k})^{p}$ with $\mathcal{B}$ a pointed Hopf algebra of dimension $4 p$. Let $\pi$ be the Hopf algebra map from $H$ onto $\mathcal{B}^{*}$. By Proposition 2.17, we have that $H^{\text {co } \pi}=\mathbb{k}\{1, x\}$ with $x$ a skew-primitive element. If $x$ is trivial, we have that $\mathbb{k} C_{2} \hookrightarrow H \rightarrow \mathcal{B}^{*}$ is an exact sequence of Hopf algebras and consequently $\mathbb{k} C_{2}$ is normal in $H$. This implies that $\mathbb{k} C_{2}$ is also normal in $\mathcal{A}$ and $\mathcal{A}$ fits into an exact sequence of Hopf algebras $\mathbb{k} C_{2} \hookrightarrow \mathcal{A} \rightarrow K$, where $\operatorname{dim} K=2 p$ so that $K$ is semisimple. Thus $\mathcal{A}$ is also semisimple, a contradiction. Therefore $x \in P_{1}(H)$ but $x \notin H_{0}$. Since $\pi(x)=0, \pi\left(P_{1}(H)\right)=0$ and in this case $\mathcal{B}^{*}$ would be the image of the coradical and hence cosemisimple, which is also a contradiction.

(ii) Suppose now that $\mathcal{A}^{*}$ is nonpointed. Recall from Subsection 2.8.1 that then $\mathcal{A} \cong \mathcal{A}(-1,1)$ and $\mathcal{A}^{*} \simeq H_{4} \oplus \mathcal{M}^{*}(2, \mathbb{k})^{p-1}$ as coalgebras. Hence $\operatorname{dim}\left(\mathcal{A}^{*}\right)_{0}=4 p-2$ and by Proposition 2.5, $\operatorname{dim}\left(H^{*}\right)_{0}=8 p-4$. Thus $H^{*}$ contains a nontrivial skew-primitive element, since otherwise Proposition 2.9 gives a contradiction. Thus $\left|G\left(H^{*}\right)\right|>1$. Since $\operatorname{dim} H^{*}-\operatorname{dim}\left(H^{*}\right)_{0}=4$ is divisible by $\left|G\left(H^{*}\right)\right|$ we have that $\left|G\left(H^{*}\right)\right|$ is 2 or 4 . But if $G\left(H^{*}\right) \cong C_{2}$ or if $G\left(H^{*}\right) \cong C_{2} \times C_{2}$, then $H^{*}$ would contain a sub-Hopf algebra isomorphic to $H_{4}$, and this is impossible by Proposition 4.6 .

Thus $H^{*}$ has a pointed sub-Hopf algebra $L$ with $G(L) \cong C_{4}$ and so $L$ has dimension 8 . Then there is a Hopf algebra epimorphism $\rho: H \rightarrow L^{*}$ and $H^{c o \rho} \cong \mathbb{k} C_{p}$ so that we have an exact sequence of Hopf algebras $\mathbb{k} C_{p} \hookrightarrow H \rightarrow L^{*}$, and dualizing we obtain the exact sequence $L \hookrightarrow H^{*} \rightarrow \mathbb{k} C_{p}$. By Proposition 2.5. $6 p=\operatorname{dim} H_{0}=p \operatorname{dim} L_{0}^{*}$. Thus we must have that $\operatorname{dim} L_{0}^{*}=6$ and $L^{*}$ cannot be pointed. We must have that $L^{*} \cong \mathcal{K}$ and $L \cong \mathcal{A}_{4}^{\prime \prime}$.

(iii) Now suppose that $\mathcal{A}^{*}$ is pointed so that $G\left(\mathcal{A}^{*}\right) \cong C_{2 p}$ by Subsection 2.8.1. Then again using Proposition 2.5 we have that $\operatorname{dim} H_{0}^{*}=4 p$. If $\left|G\left(H^{*}\right)\right| \neq 2,4$, since $H^{*}$ has a grouplike of order 2 , by Proposition 4.2 and Proposition 4.4, $\left|G\left(H^{*}\right)\right|$ must be $2 p$. Then $H_{0}^{*}=\mathbb{k} C_{2 p} \oplus E$ where $\operatorname{dim} E=2 p$ 
and $E$ is a sum of simple subcoalgebras of dimension greater than 1 . No simple subcoalgebra can have dimension divisible by $p$ since $p^{2}>2 p$. But if a simple subcoalgebra has dimension $d^{2}$ with $1<d$ and $(d, p)=1$ then $H_{0}^{*}$ must contain at least $p$ such simple coalgebras and $d^{2} p>2 p$, a contradiction.

If $H^{*}$ has a nontrivial skew-primitive element then the same argument as in (ii) above shows that $H^{*}$ has a sub-Hopf algebra isomorphic to $\mathcal{A}_{4}^{\prime \prime}$.

Corollary 4.8. If $|G(H)|=2 p$ with $p=3$ or 5 , then $H^{*}$ has a sub-Hopf algebra isomorphic to $\mathcal{A}_{4}^{\prime \prime}$.

Proof. It suffices to show that $H^{*}$ has a nontrivial skew-primitive element and then the statement follows from Proposition 4.7. We may assume that we are in case (iii) of Proposition 4.7, so that $\operatorname{dim} H_{0}^{*}=4 p$.

Let $p=3$. By Proposition [2.9, if $H^{*}$ has no skew-primitive, then for $\left|G\left(H^{*}\right)\right| \geq 2,24=\operatorname{dim} H^{*} \geq$ $12+2(5)+4=26$, a contradiction.

If $p=5$, and $\left|G\left(H^{*}\right)\right|=2$, then $\operatorname{dim} H_{0}^{*}=20$ forces $H_{0}^{*} \cong k C_{2} \oplus \mathcal{M}^{*}(3, \mathbb{k})^{2}$. Then if $H^{*}$ has no nontrivial skew-primitive, Proposition 2.9 implies that $40 \geq 20+2(7)+9=43$, a contradiction. If $p=5$ and $\left|G\left(H^{*}\right)\right|=4$, then Proposition 2.9 implies that $40 \geq 20+4(5)+4=44$, again a contradiction.

Now we can give the proof of Theorem A.

Proof of Theorem A. Let $H$ be a nonsemisimple Hopf algebra of dimension $8 p$. By Proposition 4.2 we have that $|G(H)| \in\{1,2,4,8,2 p\}$. If $|G(H)|=2 p$, then by Proposition 4.7 we have that $2 \leq\left|G\left(H^{*}\right)\right| \leq 4$ and the theorem is proved.

4.2.2. Further results for some specific primes. In this section, we improve the results of Theorem A for some specific primes $p$.

Proposition 4.9. Suppose $|G(H)|=2 p, G\left(H^{*}\right) \cong C_{4}=\langle g\rangle$ and $H^{*}$ contains a simple subcoalgebra $D$ of dimension 4. Also assume that $\left(H^{*}\right)_{0}$ is not a sub-Hopf algebra of $H^{*}$, i.e., $H^{*}$ does not have the Chevalley property. Then

(i) $D$ generates $H^{*}$ as a Hopf algebra;

(ii) $D$ is not fixed by $L_{g^{2}}, R_{g^{2}}$, i.e., by left or right multiplication by $g^{2}$. If $g^{2} \notin \mathcal{Z}\left(H^{*}\right)$ then $D$ is also not fixed by the adjoint action of $g^{2}$.

Proof. (i) Let $L=\langle D\rangle$ be the sub-Hopf algebra of $H^{*}$ generated by $D$, then $L$ is a nonpointed Hopf algebra of dimension $8,2 p, 4 p$ or $8 p$. We will show that each dimension except $8 p$ is impossible.

Suppose the dimension of $L$ is 8 . Then, by Section 4.1, either $G(L) \cong C_{2} \times C_{2}$, impossible since $G\left(H^{*}\right) \cong C_{4}$ or else $L$ contains a copy of $H_{4}$, impossible by Proposition 4.6.

Suppose the dimension of $L$ is $2 p$ so that $L \cong \mathbb{k}^{\mathbb{D}}$. Let $\mathcal{L}=\langle L, \mathbb{k}\langle g\rangle\rangle$ be the semisimple sub-Hopf algebra of $H^{*}$ generated by $L$ and by $g$, a generator of $G\left(H^{*}\right)$. Then the dimension of $\mathcal{L}$ is divisible by $2 p$ and by 4 so it must be $4 p$. Suppose that $\mathcal{L}$ is the self-dual semisimple Hopf algebra of dimension $4 p$ from Gel1] with grouplikes cyclic of order 4 . Then, if $G(H)=\langle h\rangle \cong C_{2 p}$ so that $\left\langle h^{2}\right\rangle \cong C_{p}$ and $\pi$ is the Hopf algebra projection from $H$ onto $\mathcal{L}^{*} \cong \mathcal{L}, \pi\left(h^{2 n}\right)=1$ for $0 \leq n \leq p-1$. This contradicts Lemma 2.4 which states that the dimension of $H^{c o \pi}$ is 2 .

Finally suppose that $\mathcal{L} \cong \mathbb{K}^{\Gamma}$ for $\Gamma$ a nonabelian group of order $4 p$. Then there is a Hopf algebra projection $\pi$ from $H$ onto $\mathbb{k} \Gamma$ and by Proposition $2.17, H^{c o \pi}=\mathbb{k}\{1, x\}$ where $0 \neq x$ is skew-primitive. If $x$ is trivial, then $H^{c o \pi} \cong \mathbb{k} C_{2}$. But then by Lemma 2.4 , the sequence $\mathbb{k} C_{2} \stackrel{\imath}{\hookrightarrow} H \stackrel{\pi}{\rightarrow} \mathbb{k} \Gamma$ is exact so that $H$ is semisimple, a contradiction. Thus, by the proof of Proposition 2.17, $x$ is a nontrivial $(1, b)$-primitive with $b=h$ or $b=h^{p}$ and $x^{2}=0$. Let $b=h$. Thus, since $\left\{h^{i} x \mid 0 \leq i \leq 2 p-1\right\}$ is a 
set of $2 p$ linearly independent nontrivial skew-primitive elements of $H$, all with square $0, H$ cannot have a pointed sub-Hopf algebra isomorphic to $\mathcal{A}(-1,1)$. Let $b=h^{p}$, a grouplike of order 2 . Then $H$ contains a sub-Hopf algebra isomorphic to $H_{4}$ and again cannot have a pointed sub-Hopf algebra isomorphic to $\mathcal{A}(-1,1)$. Thus $\mathcal{A}$ has pointed dual and so we are in Case (iii) of Proposition 4.7, Then $\operatorname{dim} H_{0}^{*}=4 p$ and so $\mathcal{L}=H_{0}^{*}$. Since we assumed that $H^{*}$ does not have the Chevalley property, this is a contradiction.

Suppose the dimension of $L$ is $4 p$. By its construction $L$ is not pointed. Also $L$ cannot be semisimple by the arguments in the case above where $\operatorname{dim} \mathcal{L}=4 p$. If $L$ is basic then $L \cong \mathcal{A}(-1,1)^{*} \cong H_{4} \oplus$ $\mathcal{M}^{*}(2, \mathbb{k})^{p-1}$, which is impossible since $H^{*}$ does not contain a copy of $H_{4}$. Thus both $L$ and $L^{*}$ are nonsemisimple, nonpointed so that by Theorem 2.18, $|G(L)|,\left|G\left(L^{*}\right)\right| \leq 2$. But since $L$ is a sub-Hopf algebra of $H^{*}$, we have a Hopf algebra epimorphism $\pi: H \rightarrow L^{*}$ with $\operatorname{dim} H^{\operatorname{co} \pi}=2$. This implies that $\pi\left(h^{2}\right) \neq 1$ and consequently $p \leq\left|G\left(L^{*}\right)\right| \leq 2$, a contradiction. Thus, this case is also impossible and we have proved (i), namely that $L=H$.

(ii) Now suppose that $g^{2} L=L$; if $L$ is stable under $R_{g^{2}}$ or $\operatorname{ad}_{\ell}\left(g^{2}\right)$ with $g^{2} \notin \mathcal{Z}\left(H^{*}\right)$, the argument is the same. Let $\mathcal{A} \subset H$ be the $4 p$-dimensional pointed sub-Hopf algebra of $H$ from Proposition 4.5, there is a Hopf algebra epimorphism $\pi: H^{*} \rightarrow \mathcal{A}^{*}$. If $\mathcal{A}^{*}$ is pointed, then $G\left(\mathcal{A}^{*}\right) \cong C_{2 p}$, otherwise $G\left(\mathcal{A}^{*}\right) \cong C_{2}$. In either case, $\pi\left(g^{2}\right)=1$. Then Lemma 2.14 implies that $\pi\left(H^{*}\right) \subseteq \mathbb{k} G\left(\mathcal{A}^{*}\right)$, and this contradiction finishes the proof.

Corollary 4.10. Assume $|G(H)|=2 p$ with $p=3,7,11$ and suppose that the $4 p$ dimensional pointed sub-Hopf algebra $\mathcal{A}$ of $H$ from Proposition 4.5 has pointed dual. If $H^{*}$ does not have the Chevalley property, then $G\left(H^{*}\right) \varsubsetneqq C_{4}$.

Proof. Suppose that $G\left(H^{*}\right)=\langle g\rangle \cong C_{4}$. With the notation of Proposition 4.7, the assumption that $\mathcal{A}^{*}$ is pointed means that we are in Case (iii) so that $\operatorname{dim} H_{0}^{*}=4 p$. With notation as in Proposition 4.9], it remains to show that for $p=3,7,11$, then $H^{*}$ has a simple subcoalgebra of dimension 4 stable under $L_{g^{2}}$ and that will give a contradiction.

If $p=3, H_{0}^{*} \cong \mathbb{k} C_{4} \oplus \mathcal{M}^{*}(2, \mathbb{k})^{2}$ so since the order of $g$ is 4 , the statement is clear.

If $p=7$ then either $H_{0}^{*} \cong \mathbb{k} C_{4} \oplus \mathcal{M}^{*}(4, \mathbb{k}) \oplus \mathcal{M}^{*}(2, \mathbb{k})^{2}$ or else $H_{0}^{*} \cong \mathbb{k} C_{4} \oplus \mathcal{M}^{*}(2, \mathbb{k})^{6}$ and in either case, the statement follows.

If $p=11$ then $H_{0}^{*} \cong \mathbb{k} C_{4} \oplus D$ where $D$ is one of the following: $\mathcal{M}^{*}(2, \mathbb{k})^{10}$ or $\mathcal{M}^{*}(3, \mathbb{k})^{4} \oplus \mathcal{M}^{*}(2, \mathbb{k})$ or $\mathcal{M}^{*}(4, \mathbb{k}) \oplus E$ where $E$ has dimension 24 . Then $E \cong \mathcal{M}^{*}(2, \mathbb{k})^{6}$ or $E \cong \mathcal{M}^{*}(2, \mathbb{k})^{2} \oplus \mathcal{M}^{*}(4, \mathbb{k})$. In any case, $H^{*}$ has a simple 4 -dimensional subcoalgebra stable under $L_{g^{2}}$.

Corollary 4.11. Let $\operatorname{dim} H=24$ and suppose $H$ is of type $(6,4)$ and $H^{*}$ does not have the Chevalley property. Then $H$ fits into an exact sequence $\mathcal{A}(-1,1) \hookrightarrow H \rightarrow \mathbb{k} C_{2}$, in other words, we are in Case (ii) of Proposition 4.7. Then we have that either $H_{0}^{*} \cong \mathbb{k} C_{4} \oplus \mathcal{M}^{*}(2, \mathbb{k})^{4}$ or else $H_{0}^{*} \cong \mathbb{k} C_{4} \oplus \mathcal{M}^{*}(4, \mathbb{k})$.

Proof. The statement follows from Corollary 4.8 and Corollary 4.10 ,

4.3. Generalizations of results of Cheng and $\mathbf{N g}$. In this section we generalize some results of $\mathrm{ChNg}$ to study Hopf algebras of dimension $8 p$ with group of grouplikes of order $2^{i}$. We assume throughout this section that $H$ is nonsemisimple, nonpointed, nonbasic and has dimension $8 p$.

The following propositions are similar to ChNg, Prop. 3.2].

Proposition 4.12. (i) If $H$ contains a pointed sub-Hopf algebra $K$ of dimension 8 , then $G(H)=$ $G(K)$. 
(ii) Assume $H \simeq R \# K$ where $K$ is pointed and basic of dimension 8, and $R$ is a braided Hopf algebra of dimension $p$ in $\underset{K}{K} \mathcal{Y D}$. Then $G(H) \cong G\left(H^{*}\right)$ so that $H$ is of type $(4,4)$ or type $(2,2)$.

(iii) Suppose that $|G(H)|=2^{t}$ for $t \in\{1,2,3\}$ and suppose that $H^{*}$ contains a sub-Hopf algebra $L$ of dimension 8 so that there is a Hopf algebra epimorphism $\pi: H \rightarrow L^{*}$. Then $\pi$ is an injective Hopf algebra map from $\mathbb{k} G(H)$ to $\mathbb{k} G\left(L^{*}\right)$.

(iv) Suppose that $H$ contains a pointed sub-Hopf algebra $K$ of dimension 8 with $|G(K)|=4$ and $H^{*}$ contains a pointed sub-Hopf algebra $L$ of dimension 8 . Then $K \cong L^{*}$ and $H \cong R \# K$ where $R$ is a braided Hopf algebra in ${ }_{K}^{K} \mathcal{Y} \mathcal{D}$ of dimension $p$.

Proof. (i) Suppose $H$ has a grouplike element $g$ such that $g \notin G(K)$. Then $\langle g, K\rangle$, the sub-Hopf algebra of $H$ generated by $g$ and $K$, is pointed and has dimension greater than 8 and divisible by 8 , so must be all of $H$. This is a contradiction since $H$ is not pointed.

(ii) Assume $H \simeq R \# K$ with $K$ and $K^{*}$ pointed. By (i), $G(H)=G(K)$. Since $H^{*} \simeq R^{*} \# K^{*}$, and $K^{*}$ is pointed by assumption, then again by (i), $G\left(H^{*}\right)=G\left(K^{*}\right)$. By Section 4.1, since $K$ is pointed and basic, then $G(K) \cong G\left(K^{*}\right)$ and thus $G(H) \cong G\left(H^{*}\right)$.

(iii) Dualizing the inclusion $L \subset H^{*}$, we get a Hopf algebra epimorphism $\pi: H \longrightarrow L^{*}$. Since $\operatorname{dim} L^{*}=8, \operatorname{dim} R=p$ where $R=H^{c o \pi}$ is the algebra of coinvariants. Suppose that $\pi(g)=1$ for some $g \in G(H)$ and let $\Gamma=\langle g\rangle$. Then $\mathbb{k} \Gamma \subset R$ and $R$ is a left $(H, \mathbb{k} \Gamma)$-Hopf module where the left action of $\mathbb{k} \Gamma$ on $R$ is left multiplication. Then by the Nichols-Zoeller theorem, $R$ is a free $\mathbb{k} \Gamma$-module which is impossible unless $\Gamma=\{1\}$. Thus $\pi$ is an injective Hopf algebra map on $\mathbb{k} G(H)$ as claimed.

(iv) Let $\pi: H \longrightarrow L^{*}$ and $R=H^{c o \pi}$ as in the proof of (iii). Let $x$ be a nontrivial $(g, 1)$-primitive in $K$. We wish to show that $\pi(x)$ is a nontrivial skew-primitive in $L^{*}$ and then $\pi$ will be an isomorphism from $K$ to $L^{*}$, proving the statement.

By (i), $G(H)=G(K)$ and $G\left(H^{*}\right)=G(L)$. By (iii), since $|G(H)|=4,\left|G\left(L^{*}\right)\right| \geq 4$, and since $L$ is pointed, by the description of the duals of pointed Hopf algebras of dimension 8 in Section 4.1, $L^{*}$ must also be pointed. Again, by Section 4.1, $G(L) \cong G\left(L^{*}\right)$. Let $G$ denote $G(H) \cong G(K) \cong G(L) \cong$ $G\left(L^{*}\right) \cong G\left(H^{*}\right)$.

By (iii), $\pi(x)$ is $(g, 1)$-primitive. Suppose that $\pi(K) \subseteq \mathbb{k} G \subset L^{*}$. Then $\pi(x)=\lambda(g-1)$ with $\lambda \in \mathbb{k}$. But this implies that $\pi\left(x^{2}\right)=\lambda^{2}\left(g^{2}-2 g+1\right)$, which is only possible if $\lambda=0$ since $x^{2}=0$ or $x^{2}=g^{2}-1$. Thus $\mathbb{k}\{1, x\} \subset R=H^{c o \pi}$. On the other hand, if $V$ denotes the vector space with basis $\left\{h x^{i} \mid h \in G(H), h \neq 1, i=0,1\right\}$, then $V \cap R=\{0\}$. Since $\operatorname{dim} R=p$, there is some $0 \neq z \in R$ such that $z \notin K$. Then $\langle K, z\rangle$, the sub-Hopf algebra generated by $K$ and $z$, has dimension greater than 8 and divisible by 8 so is all of $H$. By Lemma 2.3, $\pi(z) \in \mathbb{k}$. Thus $\pi(H) \subseteq \mathbb{k} G$, a contradiction, and so $\pi(z)$ is a nontrivial skew-primitive in $L^{*}$.

Corollary 4.13. Suppose that $H$ is of type $(4,4)$ and $H, H^{*}$ each have a nontrivial skew-primitive element. Then $H \cong R \# K$ where $K, K^{*}$ are pointed Hopf algebras of dimension 8 and $R$ is a braided Hopf algebra in $K_{K}^{K} \mathcal{Y D}$ of dimension $p$.

Proof. By Proposition 4.12(iv), it remains only to show that $H, H^{*}$ have pointed sub-Hopf algebras of dimension 8. Let $K=\langle G(H), x\rangle$, the sub-Hopf algebra of $H$ generated by $G(H)$ and a nontrivial skew-primitive element. Then $\operatorname{dim} K<8 p$ and is divisible by 4 so is either 8 or $4 p$. Since all pointed Hopf algebras of dimension $4 p$ have group of grouplikes of order $2 p$ (see Section 2.8.1), $\operatorname{dim} K=8$. Similarly $H^{*}$ has a pointed sub-Hopf algebra of dimension 8.

The following proposition follows the proof of $\mathrm{ChNg}$, Thm. 3.1]. 
Proposition 4.14. Let $K$ be a Hopf algebra and $R$ be a braided Hopf algebra in ${ }_{K}^{K} \mathcal{Y D}$ of odd dimension. If the order of the antipode in the bosonization $R \# K$ is a power of 2 , then $R$ and $R^{*}$ are semisimple.

Proof. Let $H=R \# K$ be the Radford biproduct or bosonization of $R$ with $K$. As $R$ is stable by $\mathcal{S}_{H}^{2}$, by [AS1, Thm. 7.3] it suffices to prove that $\operatorname{Tr}\left(\left.\mathcal{S}_{H}^{2}\right|_{R}\right) \neq 0$. Clearly, the order of $\left.\mathcal{S}_{H}^{2}\right|_{R}$ divides the order of $\mathcal{S}_{H}^{2}$ and hence is a power of 2 . If $\operatorname{Tr}\left(\left.\mathcal{S}_{H}^{2}\right|_{R}\right)=0$, then by [Ng1, Lemma 1.4] $\operatorname{dim} R$ is even, a contradiction. Thus $R$ is semisimple. The same proof holds for $R^{*}$ since $H^{*} \simeq R^{*} \# K^{*}$.

Recall that $H$ nonsemisimple of dimension 24 with $|G(H)|=4$ has a nontrivial skew-primitive element by Proposition 2.9. Then Corollary 4.13 and Proposition 4.14 imply the next statement.

Corollary 4.15. Suppose $\operatorname{dim} H=24$ and $H$ is of type $(4,4)$. Then $H \simeq R \# K$ with $K$ and $K^{*}$ pointed Hopf algebras of dimension 8 and $R$ a semisimple Hopf algebra in $K_{K}^{K} \mathcal{Y D}$ of dimension 3.

The following lemmata generalize results of Cheng and $\mathrm{Ng}$ used to study $H_{4}$-module algebras, in particular [ChNg, 3.4,3.5].

Lemma 4.16. Let $K$ be a pointed Hopf algebra generated by grouplikes and skew-primitives, and let $A$ be a finite dimensional left $K$-module algebra. If $A$ is a semisimple algebra and $e$ is a central idempotent of $A$ such that the two-sided ideal $I=$ Ae is stable by the action of $G(K)$, then $I$ is a $K$-submodule of $A$ with $g \cdot e=e$ for all $g \in G(K)$ and $x \cdot e=0$ for any skew-primitive element $x$.

Proof. Write $e=e_{1}+\cdots+e_{t}$ as a sum of orthogonal primitive central idempotents. Since $I$ is stable under the action of $G(K)$, then $G(K)$ permutes the primitive idempotents $e_{1}, \ldots, e_{t}$ and hence $g \cdot e=e$ for all $g \in G(K)$.

Let $x$ be a $(1, g)$-primitive. Then $x \cdot e=x \cdot e^{2}=(x \cdot e) e+(g \cdot e)(x \cdot e)=2(x \cdot e) e$. Thus $x \cdot e \in I$ so that $x \cdot e=(x \cdot e) e$ and then $x \cdot e=2(x \cdot e)$ implying that $x \cdot e=0$. Moreover, since $x \cdot(a e)=(x \cdot a) e$ for all $a \in A$, it follows that $I$ is stable under the action of $x$ and since $K$ is generated by grouplikes and skew-primitives, $I$ is a $K$-submodule of $A$.

Lemma 4.17. Let $K$ be a pointed Hopf algebra with abelian group of grouplikes. Let $A$ be a semisimple braided Hopf algebra in ${ }_{K}^{K} \mathcal{Y} \mathcal{D}$. If $I$ is a one-dimensional ideal of $A$, then $x \cdot I=0$ for all skew-primitive elements $x$ of $K$.

Proof. Let $x$ be a $(1, g)$-primitive element of $K$ and denote by $\bar{K}$ the pointed sub-Hopf algebra of $K$ generated by $x$ and $g$. Note that since $G(K)$ is abelian, then $g x g^{-1}=\chi(g) x=\omega x$ for some character $\chi$ of $G$ and $1 \neq \omega$ an $N$-th root of unity with $N=\operatorname{ord} g$.

Since $A$ is semisimple, $I=A e_{1}=\mathbb{k} e_{1}$ for some central primitive idempotent. Thus we need to prove that $x \cdot e_{1}=0$. If $g \cdot e_{1}=e_{1}$, then the result follows from Lemma 4.16 using $\bar{K}$ instead of $K$. Assume $g \cdot e_{1} \neq e_{1}$ and let $e_{1}, \ldots, e_{t}$ be representatives of the set $\left\{g^{i} \cdot e_{1}\right\}_{0 \leq i<N}$ of primitive central idempotents of $A$; in particular $t$ divides $N$ and $g \cdot e_{t}=e_{1}$. Let $e=e_{1}+\cdots+e_{t}$, then $\bar{I}=A e$ is a two-sided ideal of $A$ which is stable under the action of $\Gamma=\langle g\rangle$. Hence, by Lemma 4.16 we have that $x \cdot e=0$. Since $\Delta(x)=x \otimes 1+g \otimes x$, we have that $x \cdot e_{i}=x \cdot e_{i}^{2}=\alpha_{i, i} e_{i}+\alpha_{i, i+1} e_{i+1}$ and $x \cdot e_{t}=\alpha_{t, t} e_{t}+\alpha_{t, 1} e_{1}$ for some $\alpha_{i j} \in \mathbb{k}$. Using that $x \cdot e=0$ we get that $\alpha_{i-1, i}+\alpha_{i, i}=0$ and $\alpha_{t, 1}+\alpha_{1,1}=0$ for all $2 \leq i \leq t$. On the other hand, using that $\mathrm{gxg}^{-1}=\omega x$ we obtain that $\omega \alpha_{i, i}=\alpha_{i-1, i-1}$ for all $2 \leq i \leq t$, $\omega \alpha_{i, i+1}=\alpha_{i-1, i}$ for all $2 \leq i \leq t-1$ and $\omega \alpha_{1,1}=\alpha_{t, t}, \omega \alpha_{1,2}=\alpha_{t, 1}$ and $\omega \alpha_{t, 1}=\alpha_{t-1, t}$. Hence $\alpha_{1,2}=\omega^{-1} \alpha_{t, 1}=-\omega^{-1} \alpha_{1,1}$ and $x \cdot e_{1}=\alpha_{1,1}\left(e_{1}-\omega^{-1} e_{2}\right)$.

Denote by $\lambda_{A}$ the right integral of $A$. Then by [FMS, Thm. 5.8, Rmk. 5.9], see also [ChNg, Eq. (3.4)], for $k \in K, a \in A$, we have $\lambda_{A}(k \cdot a)=\varepsilon_{K}(k) \lambda_{A}(a)$. Then $g \cdot e_{1}=e_{2}$ implies that $\lambda_{A}\left(e_{1}\right)=\lambda_{A}\left(e_{2}\right)$ 
and consequently

$$
0=\varepsilon_{K}(x) \lambda_{A}\left(e_{1}\right)=\lambda_{A}\left(x \cdot e_{1}\right)=\alpha_{1,1}\left(\lambda_{A}\left(e_{1}\right)-\omega^{-1} \lambda_{A}\left(e_{2}\right)\right)=\alpha_{1,1}\left(1-\omega^{-1}\right) \lambda_{A}\left(e_{1}\right) .
$$

This implies that $\alpha_{1,1}=0$, since $\omega^{-1} \neq 1$ and $\lambda_{A}\left(e_{1}\right) \neq 0$ because the kernel of a right integral does not contain any nontrivial ideal. Hence $x \cdot e_{1}=0$ and the lemma is proved.

Proposition 4.18. Suppose that $H \cong R \# K$ where $K$ is a pointed Hopf algebra of dimension 8, and $R$ is a Hopf algebra of dimension $p$ in $K_{K}^{K} \mathcal{Y D}$ such that $x \cdot R=0$ for some $(1, g)$ primitive $x \in K, x \cdot R$ being the adjoint action of $x$ on $R$.

(i) If $|G(H)|=4$, suppose furthermore that $K$ is basic and the condition above holds for $R^{*}$ and $K^{*}$, i.e., for $y$ some nontrivial $(1, h)$-primitive in $K^{*}$, then $y \cdot R^{*}=0$. Then, $H$ and $H^{*}$ have the Chevalley property and, in the notation of Section 4.1, we have

(a) $G(H) \cong C_{4}$, and $K \cong \mathcal{A}_{4}^{\prime}$ or $K \cong \mathcal{A}_{4, \xi}^{\prime \prime \prime}$; or

(b) $G(H) \cong C_{2} \times C_{2}$ and $K \cong \mathcal{A}_{2,2}$.

(ii) If $|G(H)|=2$ then there is a Hopf algebra epimorphism $\pi: H \rightarrow A$ where $A$ is a Hopf algebra of dimension $4 p$ which is nonsemisimple, nonpointed and nonbasic. Thus if $p \leq 11$, this situation cannot occur.

Proof. We note that by Proposition 4.12(i), $G(K)=G(H)$.

(i) Let $J$ be the Hopf ideal of $H$ generated by $x$. Since $x \cdot R=0$, then $x R=-g R x \subseteq H x$ and so $J=H x$. As a $\mathbb{k}$-space, $J=\operatorname{Span}\left\{r_{i} h x: r_{1}, \ldots, r_{p}\right.$ a basis for $\left.R, h \in G(H)\right\}$ and so the dimension of $J$ is at most $4 p$. Then $\operatorname{dim} H / J \geq 4 p$ and divides $8 p$ since $(H / J)^{*}$ is isomorphic to a sub-Hopf algebra of $H^{*}$. Thus $\operatorname{dim} J=\operatorname{dim} H / J=4 p$. Then there is a Hopf algebra epimorphism $\pi: H \rightarrow A$ where $A:=H / J$ is a Hopf algebra of dimension $4 p$ and $H^{c o \pi}=\mathbb{k}\{1, x\}$. By Proposition 2.17, $x^{2}=0$ so that $K \cong \mathcal{A}_{4}^{\prime}$ or $\mathcal{A}_{4, \xi}^{\prime \prime \prime}$ if $G(H) \cong C_{4}$ and $K \cong \mathcal{A}_{2,2}$ if $G(H) \cong C_{2} \times C_{2}$. Since $\left(\mathcal{A}_{4}^{\prime}\right)^{*} \simeq \mathcal{A}_{4, \xi}^{\prime \prime \prime}$, in the former case, $H^{*} \simeq R^{*} \# \mathcal{A}_{4, \xi}^{\prime \prime \prime}$ or $R^{*} \# \mathcal{A}_{4}^{\prime}$, and since $\mathcal{A}_{2,2}$ is self-dual, we have $H^{*} \cong R^{*} \# \mathcal{A}_{2,2}$ in the later.

Since $\pi: H \rightarrow A:=H / J$ is injective on $\mathbb{k} G(H), 4$ divides $|G(A)|$. Thus, by Theorem 2.18, if $A$ is not semisimple, $A$ is pointed. But every pointed Hopf algebra of dimension $4 p$ has group of grouplikes of order $2 p$ which is not divisible by 4 , so $A$ must be semisimple.

Since $\mathcal{A}_{2,2}$ is self-dual, we have $H^{*} \cong R^{*} \# \mathcal{A}_{2,2}$. The same argument as for $H$ then gives us a Hopf algebra epimorphism from $H^{*}$ to a semisimple Hopf algebra $B$ of dimension $4 p$ with coinvariants $\{1, y\}$ where $y$ is $(1, h)$-primitive.

Then $B^{*}$ is isomorphic to a sub-Hopf algebra of $H$, call it $L$. Since $L$ is cosemisimple, $L \subseteq H_{0}$ and we wish to show equality. Since $L$ has dimension $4 p$, the sub-Hopf algebra $\langle L, x\rangle$ of $H$ generated by $L$ and $x$ is all of $H$. Since $\pi(x)=0$, this means that by dimensions $\pi$ is injective on $L$ and so $\pi: L \cong A$ is a Hopf algebra isomorphism. This implies that $H \cong S \# A$ where $S=\mathbb{k}\{1, x\}$ is a braided Hopf algebra in ${ }_{A}^{A} \mathcal{Y D}$ and thus $H$ has the Chevalley property. Reversing the roles of $H^{*}$ and $H$ in the above argument we get that $H^{*}$ also has the Chevalley property.

(ii) Now suppose that $|G(K)|=2$ and $K \cong \mathcal{A}_{2}$. Then $G(K)=\langle g\rangle$ and $K$ is generated by $g$ and two $(1, g)$-primitives, $x$ and $x^{\prime}$. Let $J$ be the Hopf ideal of $H$ generated by $x$ and as in (i), $J=H x$. Thus as a $\mathbb{k}$-space, $J=\operatorname{Span}\left\{r_{i} g^{j} z \mid r_{1}, \ldots, r_{p}\right.$ a basis for $\left.R, j=0,1, z \in\left\{x, x^{\prime} x\right\}\right\}$. Thus $\operatorname{dim} J \leq 4 p$ so that $\operatorname{dim} H / J \geq 4 p$ and is a divisor of $8 p$ so $\operatorname{dim} H / J=4 p$ and as above there is a Hopf algebra epimorphism $\pi: H \rightarrow A$ where $A:=H / J$ is a Hopf algebra of dimension $4 p$ and $H^{c o \pi}=\{1, x\}$. Since $\pi\left(x^{\prime}\right), \pi(g)$ generate a sub-Hopf algebra of $A$ isomorphic to $H_{4}$, then $A$ is not semisimple. If $A^{*}$ is pointed, then $H^{*}$ has grouplikes of order $2 p$. This is a contradiction since $H^{*} \cong R^{*} \# K^{*}$ with $K^{*} \cong \mathcal{A}_{2}$, 
so that by Proposition 4.12 (i), $G\left(H^{*}\right)=G\left(\mathcal{A}_{2}\right) \cong C_{2}$. Suppose that $A$ is pointed. Since $A^{*}$ is not pointed, then $A \cong \mathcal{A}(-1,1)$ in the notation of Section 2.8.1. But this is impossible since $\mathcal{A}(-1,1)$ has no sub-Hopf algebra isomorphic to $H_{4}$. By Theorem 2.19, for $p \leq 11, A$ is either semisimple, pointed or basic.

Corollary 4.19. Suppose $H \cong R \# K$ where $K$ is a pointed Hopf algebra of dimension 8, and $R$ is commutative and semisimple.

(i) If $|G(H)|=2$, then there is a Hopf algebra map $\pi$ from $H$ onto a Hopf algebra $A$ of dimension $4 p$ which is nonsemisimple, nonpointed and nonbasic.

(ii) If $|G(H)|=4$ and furthermore $K$ is basic and $R^{*}$ is commutative and semisimple, then $H$ and $H^{*}$ have the Chevalley property.

Proof. It remains only to show that under the given conditions there is a $(1, g)$-primitive $x$ such that $x \cdot R=0$. Since $R$ is semisimple commutative, $R$ can be written as the sum of one-dimensional simple ideals $R e_{i}$ with $e_{i}$ a central primitive idempotent. Now apply Lemma 4.17 and Proposition 4.18.

Corollary 4.20. If $\operatorname{dim} H=24$ and $H$ has type $(4,4)$, then $H$ and $H^{*}$ have the Chevalley property.

Proof. By Corollary 4.15, $H \cong R \# K$ where $K, K^{*}$ are pointed Hopf algebras of dimension $8, R$ is a semisimple braided Hopf algebra in ${ }_{K}^{K} \mathcal{Y} \mathcal{D}$ of dimension 3 , and $R^{*}$ is a semisimple braided Hopf algebra in $K_{K^{*}}^{*} \mathcal{Y D}$ of dimension 3. Since all simple representations of $R$ and $R^{*}$ must be one-dimensional, $R, R^{*}$ are commutative and the result follows from Corollary 4.19.

Remark 4.21. Suppose that $H$ is of type $\left(2^{i}, 2^{j}\right)$, has dimension 24 and $H \cong R \# K$ where $K$ is pointed of dimension 8. Then $|G(H)| \neq 2$. For by Proposition 4.14, $R$ and $R^{*}$ are semisimple and thus, since both have dimension 3, they are commutative also. Then the conditions of Proposition 4.18 hold.

The next remark summarizes the results proved for various particular dimensions $8 p$, with $H$ nonsemsimple, nonpointed, nonbasic as assumed throughout this section.

Remark 4.22. (i) From Remark 4.3, if $\operatorname{dim} H=24,40,56$, then $|G(H)| \neq 8$.

(ii) From Corollary 4.8, if $\operatorname{dim} H=24,40$, then type $(2 p, 2)$ is impossible and for type $(2 p, 4)$, $G\left(H^{*}\right) \cong C_{4}$.

(iii) From Corollary 4.10, if $p=3,7,11,|G(H)|=2 p, H^{*}$ does not have the Chevalley property, and $H$ does not contain a copy of $\mathcal{A}(-1,1)$, i.e., we are in Case (iii) of Proposition 4.7, then $G\left(H^{*}\right) \nsucceq C_{4}$.

(iv) From Corollary 4.11 if $\operatorname{dim} H=24$ and $H$ has type $(6,4)$ then if $H^{*}$ does not have the Chevalley property, then $H$ has a sub-Hopf algebra isomorphic to $\mathcal{A}(-1,1), \operatorname{dim} H_{0}^{*}=20$ and as coalgebras, either $H^{*} \cong \mathcal{A}_{4}^{\prime \prime} \oplus \mathcal{M}^{*}(2, \mathbb{k})^{4}$ or $H^{*} \cong \mathcal{A}_{4}^{\prime \prime} \oplus \mathcal{M}^{*}(4, \mathbb{k})$.

(v) By Corollary 4.20, if $\operatorname{dim} H=24$ and $H$ does not have the Chevalley property, then $H$ is not of type $(4,4)$.

4.4. Hopf algebras of dimension 24. In this subsection we specialize to the case of $p=3$, $\operatorname{dim} H=24$. Unless otherwise stated, throughout this section $H$ will denote a Hopf algebra without the Chevalley property.

Our first result is a general statement for all Hopf algebras of dimension $8 p$ and will need the following remark about nonabelian groups of order $4 p$.

Remark 4.23. Suppose that $L$ is a nonabelian group of order $4 p, p$ an odd prime. Then unless $p=3$ and $L=\mathbb{A}_{4}, L$ has a normal subgroup $N$ of order $p$. (This follows from the Sylow Theorems; see, for 
example, [L, p. 34].) Then there is a Hopf algebra map from $\mathbb{k} L$ to $\mathbb{k}(L / N)$ where $L / N$ is a group of order 4. Dualizing we see that $\mathbb{k}^{L}$ contains a sub-Hopf algebra isomorphic to a group algebra of dimension 4 and thus $G\left(\mathbb{k}^{L}\right)$ is a group of order 4 .

Proposition 4.24. Let $H$ be a nonsemisimple, nonpointed nonbasic Hopf algebra with $\operatorname{dim} H=8 p$ and suppose $H$ has a simple subcoalgebra $D$ of dimension 4 stable under the antipode. Then $H$ has a nontrivial grouplike element of order 2 .

Proof. Let $\mathcal{H}$ denote the sub-Hopf algebra of $H$ generated by $D$. Then $\operatorname{dim} \mathcal{H} \neq 2,4, p$ and so $\operatorname{dim} \mathcal{H}=$ $8,2 p, 4 p$ or $8 p$.

If $\operatorname{dim} \mathcal{H}=8$, then by the classification of Hopf algebas of dimension $8,[\mathrm{~W}],[\mathrm{S}], \mathcal{H} \cong \mathbb{k}\left[C_{2} \times C_{2}\right] \oplus$ $\mathcal{M}^{*}(2, \mathbb{k})$ as coalgebras if $\mathcal{H}$ is semisimple and $\mathcal{H} \cong H_{4} \oplus \mathcal{M}^{*}(2, \mathbb{k})$ if $\mathcal{H}$ is basic. In either case, $\mathcal{H}$, and thus $H$, contains a grouplike element of order 2 .

If $\operatorname{dim} \mathcal{H}=2 p$, then by $\mathrm{Ng} 3, \mathcal{H}$ is semisimple, so that $\mathcal{H}=\mathbb{k}^{\mathbb{D}_{p}}$ and has a grouplike of order 2 .

Now suppose that $\operatorname{dim} \mathcal{H}=4 p$. By Proposition 2.13, $\mathcal{H}$ fits into a central exact sequence:

$$
\mathbb{K}^{G} \stackrel{i}{\hookrightarrow} \mathcal{H} \stackrel{\pi}{\rightarrow} A
$$

for a group $G$ and $A$ a nonsemisimple basic Hopf algebra. Then $|G| \in\{1,2,4, p, 2 p, 4 p\}$. If $|G|=1$, then $\mathcal{H}$ is nonpointed nonsemisimple but has pointed dual, so by Subsection 2.8.1, $\mathcal{H} \cong \mathcal{A}(-1,1)^{*} \cong$ $H_{4} \oplus \mathcal{M}^{*}(2, \mathbb{k})^{p-1}$ as coalgebras and consequently has a grouplike element of order 2 . If $|G| \in\{2,4,2 p\}$, then $\mathbb{k}^{G}$ has also a grouplike element of order 2 . If $|G|=p$, then $p$ divides $|G(\mathcal{H})|$ and $|G(H)|$ so that by Proposition 4.2, $G(H) \cong C_{2 p}$ and $H$ has a grouplike of order 2 . If $|G|=4 p$, then $\mathcal{H}=\mathbb{k}^{G}$ for $G$ a nonabelian group of order $4 p$. By Remark 4.23 , $\mathbb{k}^{G}$ has a group of grouplikes of order 4 unless $p=3$, $G=\mathbb{A}_{4}$ and the dimension of $\mathcal{H}$ is 12 . But if $\mathcal{H}=\mathbb{k}^{\mathbb{A}_{4}}$ does not have a grouplike of order 2 , then as a coalgebra $\mathbb{k}^{\mathbb{A}_{4}} \cong \mathbb{k}_{3} \oplus \mathcal{M}^{*}(3, \mathbb{k})$. But $\mathcal{H}$ has a simple subcoalgebra of dimension 4 , so this case is impossible.

Finally, assume that $D$ generates $H$ so that as above, we have an exact sequence $\mathbb{K}^{G} \stackrel{i}{\hookrightarrow} H \stackrel{\pi}{\rightarrow} A$ for a group $G$ and $A$ a nonsemisimple basic Hopf algebra. Since $H$ is assumed to be nonbasic, then $|G| \neq 1$, and since $H$ is nonsemsimple, $|G| \neq 8 p$. The argument above shows that if $|G| \in\{2,4, p, 2 p\}$, then $H$ has a grouplike element of order 2. If $|G|$ is 8 or $4 p$, then $A$ has dimension $p$ or 2 respectively and so must be semisimple. This would imply that $H$ is semisimple, a contradiction.

Lemma 4.25. If $\operatorname{dim} H=24$ then $H$ has a grouplike element of order 2 .

Proof. By Proposition 4.2, $G(H) \varsubsetneqq C_{p}=C_{3}$ so it suffices to show that $H$ has a nontrivial grouplike element, i.e., that $H_{0}$ is not of the form $\mathbb{k} \cdot 1 \oplus E$ where $E$ is a sum of simple subcoalgebras of dimension greater than 1. Suppose that

$$
H_{0}=\mathbb{k} \cdot 1 \oplus \oplus_{i=1}^{t} D_{i} \text { where } D_{i} \cong \mathcal{M}^{*}\left(n_{i}, \mathbb{k}\right) \text { and } n_{j} \leq n_{j+1} .
$$

By Proposition 2.9, $\operatorname{dim} H_{0} \leq 15$ so that the possibilities for $H_{0}$ are $H_{0}=\mathbb{k} \cdot 1 \oplus \mathcal{M}^{*}(2, \mathbb{k})^{s}$ with $s=1,2,3, H_{0}=\mathbb{k} \cdot 1 \oplus \mathcal{M}^{*}(3, \mathbb{k})$ or $H_{0}=\mathbb{k} \cdot 1 \oplus \mathcal{M}^{*}(2, \mathbb{k}) \oplus \mathcal{M}^{*}(3, \mathbb{k})$. If $H$ has a simple subcoalgebra of dimension 4 stable under the antipode then by Proposition 4.24, $H$ has a grouplike element of order 2. If $H_{0}=\mathbb{k} \cdot 1 \oplus \mathcal{M}^{*}(3, \mathbb{k})$ then Proposition 2.9 implies that $\operatorname{dim} H \geq 26$, a contradiction. Thus only the cases $H_{0}=\mathbb{k} \cdot 1 \oplus \mathcal{M}^{*}(2, \mathbb{k})^{s}$ with $s=2,3$ and $S\left(D_{i}\right) \neq D_{i}$ remain.

Suppose that $H_{0}=\mathbb{k} \cdot 1 \oplus \sum_{i} D_{i}$ with $D_{i} \cong \mathcal{M}^{*}(2, \mathbb{k})$ and $S\left(D_{i}\right)=D_{j}$ for some $j \neq i$. Note that $\operatorname{dim} H_{0}>8$. Let $\mathcal{D}$ denote the set of $D_{i}$. Then since 4 divides $\operatorname{dim} D_{i}, 2 \operatorname{dim} P^{1, \mathcal{D}}$, and $\operatorname{dim} P^{\mathcal{D}, \mathcal{D}}$, then 
4 divides $1+\operatorname{dim} P^{1,1}$ and $\operatorname{dim} P^{1,1} \geq 3$. Thus by Lemma 2.6. $P_{\ell}^{1,1}$ is nondegenerate for some $\ell>2$. Then $P_{m}^{1, D_{i}}, P_{1}^{D_{i}, 1}, P_{1}^{1, S\left(D_{i}\right)}$ are nondegenerate for $m=\ell-1 \geq 2$, some $i$. Then $2 \operatorname{dim} P^{1, \mathcal{D}} \geq 8$. Since $P_{1}^{D_{i}, 1}$ and $P_{m}^{S\left(D_{i}\right), 1}$ are nondegenerate then $P^{D_{i}, \mathcal{D}}$ and $P^{S\left(D_{i}\right), \mathcal{D}}$ are nondegenerate and $\operatorname{dim} P^{\mathcal{D}, \mathcal{D}} \geq 8$. But this is impossible if $\operatorname{dim} H=24$.

Remark 4.26. Similar arguments to the proof of Lemma 4.25 apply if $\operatorname{dim} H=4 n$ and $H_{0}=\mathbb{k}$. $1 \oplus \sum_{i=1}^{t} D_{i}$ with $D_{i}=\mathcal{M}^{*}(2, \mathbb{k})$ and $D_{i} \neq S\left(D_{i}\right)$ for all $i$. Let $\mathcal{D}$ denote the set of $D_{i}$. Then $2 \operatorname{dim} P^{1, \mathcal{D}}+\operatorname{dim} P^{\mathcal{D}, \mathcal{D}} \geq 20$ where $\mathcal{D}$ denotes the set of simple 4-dimensional subcoalgebras.

For, we may suppose that $P^{1,1}=P_{\ell}^{1,1}$ with $\ell \geq 3$. Then $P_{1}^{1, C}, P_{\ell-1}^{C, 1}, P_{1}^{S(C), 1}, P_{\ell-1}^{C, E}, P_{\ell-2}^{C, D}, P_{1}^{D, 1}$ are nondegenerate for some $C, D, E \in \mathcal{D}$ so that $2 \operatorname{dim} P^{1, \mathcal{D}} \geq 8$ and $\operatorname{dim} P^{\mathcal{D}, \mathcal{D}} \geq 8$. Furthermore, since $\ell-1 \geq 2$, then $P_{1}^{C, X}, P_{\ell-2}^{X, E}$ are nondegenerate for some coalgebra $X$. If $\operatorname{dim} X=1$, then $P_{1}^{C, 1}, P_{1}^{S(C), 1}, P_{\ell-1}^{C, 1}$ are nondegenerate and $2 \operatorname{dim} P^{1, \mathcal{D}} \geq 12$. If $\operatorname{dim} X=4$, then $P_{1}^{C, X}, P_{\ell-1}^{C, E}, P_{\ell-2}^{C, D}$ are nondegenerate and the statement follows.

We finish the section with the proof of Theorem B.

Proof of Theorem B. Let $\operatorname{dim} H=24$ and suppose that $H$ does not have the Chevalley property. Then $|G(H)| \neq 1,3,8,12$ or 24 , by Lemma 4.25, Remark 4.3 and Proposition 4.2 . Since $|G(H)|$ divides $\operatorname{dim} H$, we have that $|G(H)| \in\{2,4,6\}$ and by Remark 4.22, the proof is complete. 


\section{OPEN CASES}

The following table enumerates all open cases in the classification of Hopf algebras of dimension less than 100 up to isomorphism. In this table, $p$ is arbitrary, not necessarily odd.

\begin{tabular}{|c|c|c|c|c|}
\hline $\operatorname{dim} H$ & Semisimple & Pointed & Chevalley & Other \\
\hline$p$ & $\begin{array}{l}\text { Completed: } \\
\text { All trivial [Z] }\end{array}$ & None & None & None: [Z] \\
\hline $\begin{array}{l}2 p \\
p \text { odd }\end{array}$ & $\begin{array}{l}\text { Completed: } \\
\text { All trivial Mas5 } 1\end{array}$ & None & None & None: Ng3 \\
\hline$p^{2}$ & $\begin{array}{l}\text { Completed: All trivial } \\
\text { Mas3 }\end{array}$ & $\begin{array}{l}\text { Completed: } \exists p-1 \text {, the Taft } \\
\text { Hopf algebras AS1 }\end{array}$ & None & None: Ng1 \\
\hline$p q$ & $\begin{array}{l}\text { Completed: All trivial } \\
\begin{array}{|l|l|l|}\text { Mas2 } & \text { Ng3 } & \text { EGel1 } \\
\text { GelW } & \text { So } & \text { N2 } \\
\end{array} \\
\end{array}$ & None & None & $\begin{array}{l}\text { None: for } p<q \leq 4 p+11 \\
\text { Ng4 } \\
\text { Open: } 87,93 .\end{array}$ \\
\hline$p^{3}$ & $\begin{array}{l}\text { Completed: } \\
p=2, \exists 1[\text { KacP }[\text { Mas } 2] \\
p \text { odd }, \exists p+1[\text { Mas4 }]\end{array}$ & $\begin{array}{l}\text { Completed: } p=2, \exists 5 \text { [S] } \\
p \text { odd } \exists(p-1)(p+9) / 2 \text { AS2 } \\
\begin{array}{ll}\text { CD }[\text { ŞvO }\end{array}\end{array}$ & None & $\begin{array}{l}\text { None : } \\
8[\mathrm{~W}],[\mathrm{S}] \\
27[\mathrm{G}],[\mathrm{BG}]\end{array}$ \\
\hline $\begin{array}{l}2 p^{2} \\
p \text { odd }\end{array}$ & $\begin{array}{l}\text { Completed: } \exists 2 \text {, they } \\
\text { are duals [Mas1], N1] }\end{array}$ & $\begin{array}{l}\text { Completed: } \\
\exists 4(p-1) \text { [AN1, A.1] }\end{array}$ & None & None $\sqrt[2]{\mathrm{HNg}}$ \\
\hline $\begin{array}{l}p q^{2} \\
p \text { odd }\end{array}$ & 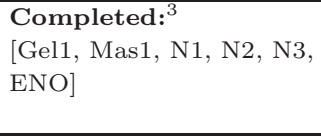 & $\begin{array}{l}\text { Completed: } \\
\exists 4(q-1) \text { AN1 A.1] }\end{array}$ & None: AN1 Lemma A.2] & $\begin{array}{l}\text { Completed: } 12 \text { N5 } \\
20,28,44 \text { ChNg } \\
\text { Open: } 45,52,63,68,75 \text {, } \\
76,92,99 .\end{array}$ \\
\hline$p q r$ & $\begin{array}{l}\text { Completed:4 } \\
\text { [N1, N2] ENO] } \\
\end{array}$ & None & None: $\quad$ Prop. 3.2 & $\begin{array}{l}\text { Completed: } 30[\mathrm{Fu} 3 \\
\text { Open: } 42,66,70,78\end{array}$ \\
\hline$p^{4}$ & $\begin{array}{l}\text { Completed: } p=2, \exists 16 \\
\text { K, Theorem 1.2] } \\
\text { Open: } 81\end{array}$ & $\begin{array}{l}\text { Completed: } 16 ; \exists 29 \text { CDR } 5 \\
\text { Completed: } p \text { odd [AS2]. Infi- } \\
\text { nite nonisomorphic families ex- } \\
\text { ist [AS2], [BDG], [Gel2] }{ }^{10}\end{array}$ & $\begin{array}{l}\text { Completed: } 16[\text { CDMM } \\
\exists 2 \text { selfdual, coradical } A_{8} \\
\text { Open: } 81\end{array}$ & $\begin{array}{l}\text { Completed: } 16 \mathrm{GV} \\
\text { Open: } 81\end{array}$ \\
\hline$p^{3} q$ & Open & $\begin{array}{l}\text { Completed } 16 \\
24,40,54,56 \text { Gr1 } \\
\text { Open: } 88^{9}\end{array}$ & Open & $\begin{array}{l}\text { Open: } \\
24,40,54,56,88\end{array}$ \\
\hline$p^{2} q^{2}$ & Open & $\begin{array}{l}\text { Completed: } 36 \text { Gr1] } \\
\text { Open: } 100^{9}\end{array}$ & Open & Open: 36,100 \\
\hline$p^{2} q r$ & Open & $\begin{array}{l}\text { Completed: } 60 \text { Gr1 } \\
\text { Open: } 84,90^{9}\end{array}$ & Open & Open: $60,84,90$ \\
\hline$p^{3} q^{2}$ & Open & Open $\sqrt{7}$ & Open $\$$ & Open: 72 \\
\hline $\begin{array}{l}p^{n} \\
n=5,6\end{array}$ & Open & $\begin{array}{l}\text { Completed: } 32 . \mathrm{Gr} 2 \text { Infinite } \\
\text { families of nonisomorphic Hopf } \\
\text { algebras exist. Gr2, } \text { [B1 }^{10} \\
\text { Open: } 64\end{array}$ & Open & Open: 32,64 \\
\hline$p^{4} q$ & Open & $\begin{array}{l}\text { Completed: } 48 \text { Gr1] } \\
\text { Open: } 80\end{array}$ & Open & Open: 48,80 \\
\hline$p^{5} q$ & Open & Open $^{6}$ & Open & Open: 96 \\
\hline
\end{tabular}

TABLE 1. Hopf algebras of dimension $\leq 100$

${ }^{1}$ Dimension 6 was classified in Mas2.

${ }^{2}$ The classification for dimension $18=2\left(3^{2}\right)$ was completed in Fu1.

${ }^{3}$ The complete classification of semisimple Hopf algebras of dimension $12=3\left(2^{2}\right)$ is given in $\mathbf{F}$.

${ }^{4}$ The complete classification of semisimple Hopf algebras of dimension 30 and 42 is given in [N4].

${ }^{5}$ The duals to these are explicitly constructed in [B3].

${ }^{6}$ Pointed Hopf algebras $H$ with $\frac{\operatorname{dim} H}{|G(H)|}<32$ or $\frac{\operatorname{dim} H}{|G(H)|}=p^{3}$ were classified in [Gr1].

${ }^{7}$ Pointed Hopf algebras with nonabelian grouplikes known to exist by AHS dimension $p^{3} p^{2}$, [FG] dimension $p^{5} q$.

${ }^{8}$ Nonpointed Hopf algebras with Chevalley property known to exist AV1, AV2.

${ }^{9} \operatorname{dim} p^{3} q, p^{2} q^{2}, p^{2} q r$ : For dimensions 88, 100, 84, 90, the classification of the pointed Hopf algebras was completed for those with coradical a group algebra of order a power of 2 in [Ni] and Gr1].

${ }^{10}$ The families of nonisomorphic pointed Hopf algebras of dimension 81 consist of quasi-isomorphic Hopf algebras Mas6] but the duals of the families of nonisomorphic pointed Hopf algebras of dimension 32 give an infinite family of non-quasi-isomorphic Hopf algebras EGel2. 
The columns from left to right describe the classification of Hopf algebras which are semisimple, pointed nonsemisimple, nonsemisimple nonpointed with the Chevalley property, etc. We call a Hopf algebra trivial if it is a group algebra or the dual of a group algebra. For dimension $m n^{2}$, pointed Hopf algebras always exist; just take $\mathbb{k} C_{m} \otimes T_{q}$ where $q$ is a primitive $n$th root of unity.

Note that by [AN1, Prop. 1.8], a Hopf algebra of square-free dimension cannot be pointed. Also note if for every divisor $m$ of some dimension $n$ the only semisimple Hopf algebras of dimension $m$ are the group algebras, then there are no Hopf algebras of dimension $n$ with the Chevalley property. For example, this is why there are no nonpointed Hopf algebras of dimension $p^{3}$ with the Chevalley property.

Examples of nonpointed but basic Hopf algebras do exist. They are given by duals of nontrivial liftings which are not Radford bosonizations. See for example [B2].

In general, this table does not contain references to partial results for a particular dimension even though the literature may contain some. For example the general classification for dimension 24 is listed only as Open. Also when a general result has been proven, the table cites only that result. For example, $\mathrm{HNg}$ is cited for the result that all Hopf algebras of dimension $2 p^{2}, p$ odd, are semisimple or pointed; the specific case of dimension 18 was proved in Fu1. We have attempted to include references to some specific cases in the footnotes but make no claim that these are complete.

\section{ReFEREnCES}

[AD] N. Andruskiewitsch and J. Devoto J, Extensions of Hopf algebras, Algebra i Analiz 7, No. 1 (1995), 22-61; translation in St. Petersburg Math. J. 7 (1996), no. 1, 17-52.

[AEGel] N. Andruskiewitsch, P. Etingof and S. Gelaki, Triangular Hopf algebras with the Chevalley property, Michigan Math. J. 49, no. 2 (2001), 277-298.

[AHS] N. Andruskiewitsch, I. HeCkenberger and H.-J. Schneider, The Nichols algebra of a semisimple YetterDrinfeld module, Amer. J. Math., vol. 132, no. 6, December 2010, 1493-1547.

[AN1] N. Andruskiewitsch and S. Natale, Counting arguments for Hopf algebras of low dimension, Tsukuba Math J. 25, No. 1 (2001), 187-201.

[AN2] Examples of self-dual Hopf algebras, J. Math. Sci. Univ. Tokyo 6 (1999), 181-215.

[AS1] N. Andruskiewitsch and H.-J. Schneider, Hopf algebras of order $p^{2}$ and braided Hopf algebras of order $p$, J. Algebra 199 (1998), no. 2, 430-454.

[AS2] _ Lifting of quantum linear spaces and pointed Hopf algebras of order $p^{2}$, J. Algebra 209 (1998), no. 2, $658-691$.

[AV1] N. Andruskiewitsch and C. VAY, Finite dimensional Hopf algebras over the dual group algebra of the symmetric group in three letters, Comm. Algebra 39 (2011), 4507-4517.

[AV2] - On a family of Hopf algebras of dimension 72, Bulletin of the Belgian Mathematical Society, to appear. Preprint: arXiv:1105.0394.

[B1] M. BeAtTie, An isomorphism theorem for Ore extension Hopf algebras, Comm. Algebra, 28 (2) (2000) $569-584$.

[B2] _ Duals of pointed Hopf algebras, J. Algebra 262 (2003), 54-76. Corrigendum J. Algebra 320 (2008), 3849 .

[B3] A survey of Hopf algebras of low dimension, Acta Appl. Math. 108 (2009), no. 1, 19-31.

[BD] M. BeAttie and S. DĂSCĂLESCU, Hopf algebras of dimension 14, J. London Math. Soc. II Ser. 69, No.1 (2004), 65-78.

[BDG] M. Beattie, S. DĂSCĂLescu and L. Grünenfelder, On the number of types of finite dimensional Hopf algebras, Invent. Math. 136 1-7, 1999.

[BG] M. BeAtTiE and G.A. GarcíA, Techniques for classifying Hopf algebras and applications to dimension $p^{3}$, Comm. Algebra, to appear.

[CD] S. Caenepeel and S. Dascalescu, Pointed Hopf algebras of dimension p $p^{3}$, J. Algebra 209 (1998), no. 2, 622-634.

[CDR] S. Caenepeel, S. DăscăLescu and Ş. Raianu, Classifying pointed Hopf algebras of dimension 16, Comm. Algebra 28, No. 2 (2000), 541-568. 
[CDMM] C. Calinescu, S. Dăscălescu, A. Masuoka, C. Menini, Quantum lines over non-cocommutative cosemisimple Hopf algebras, J. Algebra 273 (2004), 753-779.

[ChNg] Yi-lin Cheng and Siu-Hung NG, On Hopf algebras of dimension 4p, J. Algebra 328 (2011), 399-419.

[EGel1] P. Etingof and S. Gelaki, Semisimple Hopf algebras of dimension pq are trivial, J. Algebra 210 (1998), 664-669.

[EGel2] _ On families of triangular Hopf algebras, Int. Math. Res. Not. IMRN 14(2002), 757-768.

[EGel3] _ On Hopf algebras of dimension pq, J. Algebra 277, no. 2 (2004), 668-674.

[ENO] P. Etingof, V. Nikshych and V. Ostrik, Weakly group-theoretical and solvable fusion categories, Advances in Mathematics, 226 (2011), no. 1, 176-205.

[FG] F. Fantino and G. A. García, On pointed Hopf algebras over dihedral groups, Pacific J. Math, Vol. 252 (2011), no. 1, 69-91.

[FMS] D. Fischman, S. Montgomery and H.-J. Schneider, Frobenius extensions of subalgebras of Hopf algebras, Trans. Amer. Math. Soc. 349 (1997), no. 12, 4857-4895.

[F] N. FukudA, Semisimple Hopf algebras of dimension 12, Tsukuba J. Math. 21 (1997), 43-54.

[Fu1] D. FukudA, Classication of Hopf algebras of dimension 18, Israel J. Math. 168 (2008), 119-123.

[Fu2] Structure of coradical filtration and its application to Hopf algebras of dimension pq. Glasg. Math. J. 50 (2008), no. 2, 183-190.

[Fu3] _ Hopf algebras of dimension 30, J. Algebra Appl. 9 (2010), no. 1, 11-15.

[G] G. A. García, On Hopf algebras of dimension p $p^{3}$, Tsukuba J. Math. 29 (2005), no. 1, $259-284$.

[GV] G. A. García and C. VAY, Hopf algebras of dimension 16, Algebr. Represent. Theory, 13 (2010), $383-405$.

[Gel1] S. Gelaki, Quantum groups of dimension pq², Israel J. Math. 102 (1997), 227-267.

[Gel2] Pointed Hopf algebras and Kaplansky's 10th conjecture, J. Algebra 209, (1998) 635-657 .

[GelW] S. Gelaki and S. Westreich, On semisimple Hopf algebras of dimension pq, Proc. Am. Math. Soc. 128 (2000), 39-47. (Corrigendum: Proc. Am. Math. Soc. 128 (2000), 2829-2831.)

[Gr1] M. GrañA, On Nichols algebras of low dimension, Contemp. Math. 267 (2000), 111-134.

[Gr2] - Pointed Hopf algebras of dimension 32, Comm. Algebra 28 (6) (2000), 2935-2976.

[HNg] M. Hilgemann and S.-H. NG, Hopf algebras of dimension 2p2 , J. Lond. Math. Soc. (2) 80 (2009), no. 2, $295-310$.

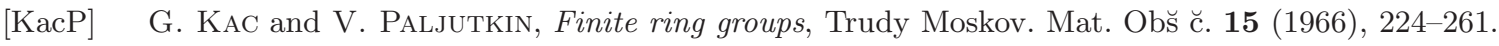

[K] Y. Kashina, Classification of semisimple Hopf algebras of dimension 16, J. Algebra 232 (2000), 617-663.

[KMM] Y. Kashina, G. MASOn and S. Montgomery, Computing the Frobenius-Schur indicator for abelian extensions of Hopf algebras, J. Algebra 251 (2002), 888-913.

[L] S. LANG, Algebra. Revised third edition. Graduate Texts in Mathematics, 211. Springer-Verlag, New York, 2002 .

[LR] R. LARSON and D. RADFORD, Finite dimensional cosemisimple Hopf algebras in characteristic 0 are semisimple, J. Algebra 117 (1988), 267-289.

[Mas1] A. MASUOKA, Some further classification results on semisimple Hopf algebras, Comm. Algebra 24 (1996), 307-329.

[Mas2] _ Semisimple Hopf algebras of dimension 6, 8, Israel J. Math. 92 (1995), 361-373.

[Mas3] - The $p^{n}$ theorem for semisimple Hopf algebras, Proc. Amer. Math. Soc. 124 no. 3 (1996), $735-737$.

[Mas4] _ Self dual Hopf algebras of dimension $p^{3}$ obtained by extension, J. Algebra 178 (1995), 791-806.

[Mas5] _ Semisimple Hopf algebras of dimension 2p, Comm. Algebra 23 (1995), 1931-1940.

[Mas6] _ _ Defending the negated Kaplansky conjecture, Proc. Amer. Math. Soc. 129 (11), (2001), 3185-3192.

[M] S. Montgomery, Hopf Algebras and their Actions on Rings, CMBS Reg. Conf. Ser. in Math. 82, Amer. Math. Soc., 1993.

[N1] S. NATALE, On semisimple Hopf algebras of dimension pq ${ }^{2}$, J. Algebra 221 (1999), 242-278.

[N2] - On semisimple Hopf algebras of dimension pq ${ }^{2}$, II, Algebr. Represent. Theory 5 (3), (2001), $277-291$.

[N3] _ On semisimple Hopf algebras of dimension pq ${ }^{r}$, Algebr. Represent. Theory 7 (2) (2004), $173-188$.

[N4] _ Semisolvability of semisimple Hopf algebras of low dimension, Mem. Amer. Math. Soc., 186 (2007), no. 874 .

[N5] Hopf algebras of dimension 12, Algebr. Represent. Theory 5, No. 5 (2002), 445-455. 
[Ng1] S-H. Ng, Non-semisimple Hopf algebras of Dimension p², J. Algebra 255, no. 1 (2002), $182-197$.

[Ng2] Hopf algebras of dimension pq, J. Algebra 276, no. 1 (2004), 399-406.

[Ng3] _ Hopf algebras of dimension 2p, Proc. Amer. Math. Soc. 133, no. 8 (2005), $2237-2242$ (electronic).

[Ng4] Hopf algebras of dimension pq, II, J. Algebra 319, no. 7 (2008), 2772-2788.

[Ni] W. Nichols, Bialgebras of type one. Comm. Algebra 6, no. 15 (1978), 1521-1552.

[So] Y. SommerhäUsen, Yetter-Drinfel'd Hopf algebras over groups of prime order, Lecture Notes in Mathematics, 1789. Springer-Verlag, Berlin, 2002. iv+158 pp.

[S] D. Ştefan, Hopf algebras of low dimension, J. Algebra 211 (1999), 343-361.

[ŞvO] D. Stefan and F. VAn Oystaeyen, Hochschild cohomology and coradical filtration of pointed coalgebras: Applications, J. Algebra 210 (1998), no. 2, 535-556.

[S] M. SweEdLer, Hopf algebras, Benjamin, New York, (1969).

[W] R. Williams, Finite dimensional Hopf algebras, Ph. D. Thesis, Florida State University (1988).

[Z] Y. ZHU, Hopf algebras of prime dimension, Int. Math. Res. Not. 1 (1994), 53-59.

Mount Allison University

SACKVILle, NB E4L 1E6

CANADA AND

Facultad de Matemática, Astronomía y Física,

Universidad Nacional de Córdoba. Ciem - CONiCET.

Medina Allende S/N

(5000) Ciudad Universitaria, Córdoba, Argentina

E-mail address: mbeattie@mta.ca

E-mail address: ggarcia@famaf.unc.edu.ar 